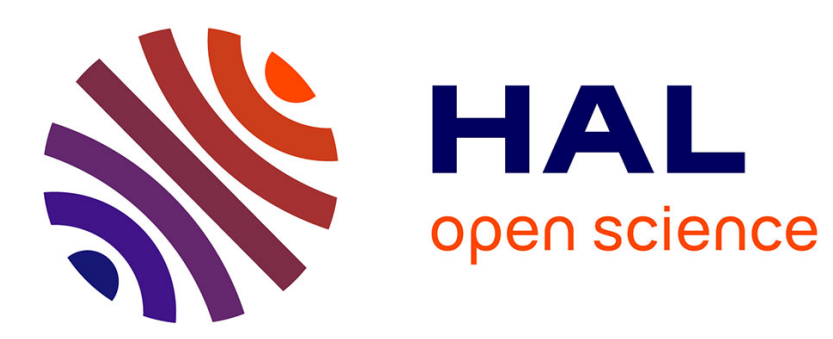

\title{
Influence of the temperature on the opto-acoustophoretic effect
}

Gabriel Dumy, Mauricio Hoyos, Jean-Luc Aider

\section{To cite this version:}

Gabriel Dumy, Mauricio Hoyos, Jean-Luc Aider. Influence of the temperature on the optoacoustophoretic effect. Journal of the Acoustical Society of America, 2021, 149 (1), pp.556-568. 10.1121/10.0003058 . hal-03433272

\section{HAL Id: hal-03433272 \\ https://hal.science/hal-03433272}

Submitted on 17 Nov 2021

HAL is a multi-disciplinary open access archive for the deposit and dissemination of scientific research documents, whether they are published or not. The documents may come from teaching and research institutions in France or abroad, or from public or private research centers.
L'archive ouverte pluridisciplinaire HAL, est destinée au dépôt et à la diffusion de documents scientifiques de niveau recherche, publiés ou non, émanant des établissements d'enseignement et de recherche français ou étrangers, des laboratoires publics ou privés. 


\title{
Influence of the temperature on the opto-acoustophoretic effect
}

\author{
Gabriel Dumy, ${ }^{1, \text { a) }}$ Mauricio Hoyos, ${ }^{1}$ and Jean-Luc Aider ${ }^{1}$ \\ Laboratory PMMH, ESPCI Paris PSL, Sorbonne Université
}

Opto-acoustophoretic mobility has been demonstrated recently for fluorescent and colored particles acoustically levitated in a stationary ultrasonic field, when illuminated with the appropriate optical wavelength ${ }^{1,2}$. It is a repeatable phenomenon, needing both acoustic trapping and specific optic excitation to occur. However, the physical origin of the phenomenon is still debated. In this study, we provide more insights into the probable origin of this phenomenon, by confronting numerical simulations with temperature controlled experiments. The phenomenon properties are well reproduced by our model relying on a thermofluidic instability, hinting at the potential thermally-induced fluid density gradient as a drag source for the observed ejection of particles. Thermostated experiments exhibit a surprising threshold, above which the phenomenon is not observed anymore, no matter how large the optic or acoustic energies used. This exciting new observation differs from the initial interpretation of the phenomenon, altering its potential application without removing its interest, since it suggests the possible contact-less generation of customized flows by acoustically trapped particles.

a) Also at Paris Descartes, University de Paris; dumy.gabriel@gmail.com 
JASA/Influence of the temperature on the opto-acoustophoretic effect

\section{INTRODUCTION}

Waves can exert forces on scattering objects. This can be observed experimentally every time a comet crosses our skies, its tail effectively blown away by the radiative pressure exerted on these lone bodies by the electromagnetic waves coming from the sun, showing avid eyes that invisible things indeed could act on the material world. Ever since, evidence after evidence has been brought up by research on electromagnetic waves, eventually producing extremely useful techniques for life sciences such as optical tweezers ${ }^{3,4}$. More recent works have focused on the forces stemming from acoustic waves, and a large amount of research is dedicated to reach the potential already achieved by optical tweezers with their acoustic counterpart $^{5-7}$.

It is quite usual now to use surface acoustic waves (SAW) or bulk acoustic waves (BAW) to build acoustic sorting devices or traps. The migration of acoustic-scattering particles in a standing acoustic wave, or acoustophoresis, has been used for over a decade to trap spherical particles in very specific locations of space, in order to study their mechanical properties ${ }^{8-10}$, and high hopes are given to grow cellular cultures while in acoustic levitation ${ }^{11-14}$. While acoustofluidics has been extensively explored in standard conditions, especially when applied to spherical rigid particles, there are still unknown phenomena discovered when applying acoustofluidics to other objects or in non-standard conditions. For instance, when the spherical symmetry assumption of the manipulated objects is no longer satisfied, such as with disks $^{15}$ or metallic micro-cylinders ${ }^{16-20}$, particles are actively propelled by the acoustic forces instead of quietly aggregating into stable positions. Unexpected effects can also arise when 
using particles with specific properties, such as hollow core particles ${ }^{21,22}$ or specific optical properties $^{1}$, which is the present study object.

The usual expression for the acoustic radiation force (ARF) experienced by spherical particles suspended in a liquid medium in a purely transverse standing wave ultrasonic acoustic field, along the direction of propagation of said field, can be derived following the Gor'kov approach ${ }^{23}$ :

$$
\boldsymbol{F}_{a c}(z)=\frac{\pi}{2}\left\langle E_{a c}\right\rangle k d_{p}^{3} F_{Y} \sin \left(2 k_{a c} z\right) \boldsymbol{e}_{z}
$$

where $\langle-\rangle$ denotes time averaging, $d_{p}$ is the particle diameter, $\left\langle E_{a c}\right\rangle$ is the time-averaged acoustic energy density inside the channel, $k=\frac{2 \pi}{\lambda_{a c}}=\frac{2 \pi f_{a c}}{c}$ is the wave number of the acoustic plane wave of frequency $f_{a c}$, and $z$ is the axial (or vertical) position of the particle, $z=0$ being at the bottom of the channel and $z=h$ being at the top. This expression can be corrected for fluid viscosity or particle elasticity ${ }^{24-26}$. However, we will neglect fluid viscosity corrections, since the particles in this study are always 5 micrometer in diameter or more, much larger than the viscosity length of $400 \mathrm{~nm}$ (at $20^{\circ} \mathrm{C}$ for a $2.0 \mathrm{MHz}$ acoustic wave in water).

The acoustic contrast factor $F_{Y}$ of a given particle of density $\rho_{p}$ in a medium of density $\rho_{f}$, exposed to an acoustic wave propagating at $c_{f}$ in the medium and at $c_{p}$ in the particle, is then defined as:

$$
F_{Y}=\frac{1+\frac{2}{3}\left(1-\frac{\rho_{f}}{\rho_{p}}\right)}{2+\frac{\rho_{f}}{\rho_{p}}}-\frac{\rho_{f} c_{f}^{2}}{3 \rho_{p} c_{p}^{2}}
$$


JASA/Influence of the temperature on the opto-acoustophoretic effect

Depending on the sign of the acoustic contrast factor, the suspended objects will move toward the pressure node or pressure anti-node of the acoustic field. Negative contrast factors object can be found amongst bubbles, or specific lipid droplets for instance. In this study we consider only objects with positive contrast factors (such as plastic particles or red blood cells), indeed moved toward the pressure node (Fig. 1a) where they will be kept in acoustic levitation (Fig. 1b and c). The particles equilibrium position is called the levitation plane, and is a result of the competition between buoyancy, gravity and acoustic forces ${ }^{27}$.

The radial component of the ARF may be neglected in many applications like acoustic sorting because it is usually one order of magnitude weaker than the axial component ${ }^{28,29}$. Nevertheless it plays a crucial role in closed cavities, once the particles have reached the levitation plane. Following ${ }^{7}$, the transverse component of the ARF inside the levitation plane can be expressed as:

$$
\boldsymbol{F}_{T}(x, y)=d_{p}^{3} \frac{3\left(\rho_{p}-\rho_{f}\right)}{\rho_{f}+2 \rho_{p}} \boldsymbol{\nabla}\left\langle E_{a c}\right\rangle(x, y)
$$

This component depends directly upon the radial gradient of the acoustic energy $\nabla\left\langle E_{a c}\right\rangle(x, y)$ and is responsible for the aggregation of particles toward the local maximum of acoustic energy in the levitation plane (Fig. 1c). It is mainly defined by the shape of the acoustic energy density landscape, which is related to the source of the ultrasonic wave and to the geometry, materials and boundary conditions of the resonant cavity.

These equations describe correctly the forces applied to spherical particles made of soft or rigid materials, such as polymers, silica, or biological materials, either passive (e.g. red blood cells), or active (e.g. bacteria ${ }^{14}$ that can extract and convert chemical energy available 
(a)

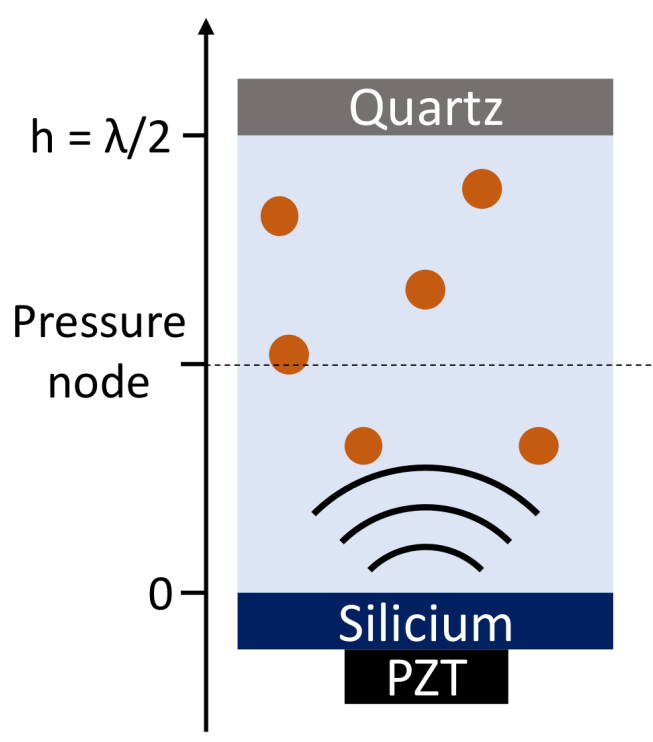

(b)

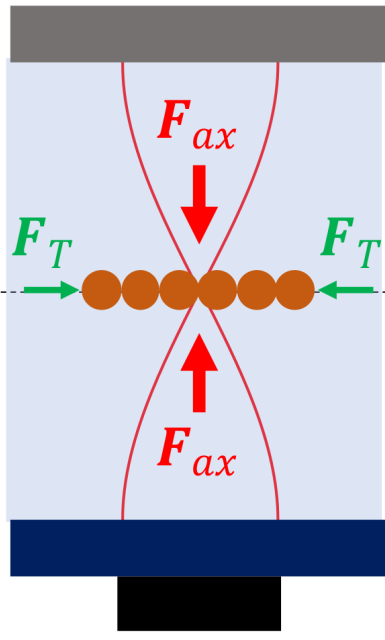

(c)

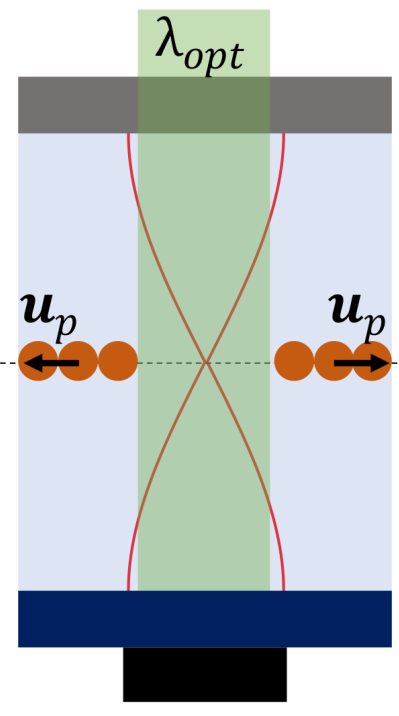

FIG. 1. (a) Sketch of the acoustic resonant cavity filled with a micrometric particle suspension (side view). A silicium wafer with a piezoelectric transducer attached makes the cavity floor, while the top wall is a quartz lid allowing good optical access for both observation and illumination. If the cavity height and the acoustic frequency are properly tuned, then the ultrasounds emitted by the transducer will build a standing wave profile, with a pressure node at cavity mid-height. (b) The particles gather at the pressure node under the ARF (both components illustrated) where they can be kept in acoustic levitation, away form the walls. (c) If the particles are enlightened with the proper optical wavelength, they can be laterally expelled from the illuminated area, while still kept in acoustic levitation.

to them into motion or forces, i.e. kinetic and potential energies). Recently, it has been shown that the acoustic focusing and aggregation process of passive particles trapped in an ultrasonic field could be affected by an illumination at specific optical wavelength ${ }^{1}$. Instead 
of being trapped and aggregated into a stable position of space (acoustic trapping), the levitated particles, if enlightened with a proper wavelength, could be radially expelled from their equilibrium aggregation position, while staying confined into the levitation plane (Fig. 1d). The phenomenon is robust and has been demonstrated for different types of fluorescent or coloured particles. A clear dependency to the amplitude of the acoustic wave as well as to the power of the light source has been found. The coupling of both actuation is needed. Different hypotheses have been raised but no definitive explanation could be assessed.

The objective of this study is to investigate a thermal origin of the phenomenon. After a short description of the experimental setup, we will present an temperature controlled experiment to assess an idea inspired by a recent extension of the classical acoustophoresis development to fluids particles ${ }^{30}$. Finally, since the main argument of this explanation rises from a thermo-acoustofluidic effect, numeric simulations are presented, enriching the discussion about the optoacoustofluidic effect generation.

\section{MATERIALS AND METHODS}

\section{A. Acoustic cavity}

In this study we use the same circular aluminium acoustic cavities as the one used in the previous study ${ }^{1}$. It consists of an in-house aluminum body, holding the different components of the device and constituting the walls of the resonant cavity. The inner dimensions of the cavity are defined by its diameter $D=20 \mathrm{~mm}$ and its height $h=800 \mu \mathrm{m}$. The height can be tuned to match the half-wavelength of the acoustic field $\lambda_{a c}=2 h$ (see Fig. 2 a-c). 
In this setup, the resonant frequency maximizing the acoustic effects is found to be around $f_{a c} \simeq 737 \mathrm{kHz}$ under ambient conditions. The lower wall of the cavity is a silicone wafer (thickness $h_{\text {trans }}=0.30 \mathrm{~mm}$ ), onto which is glued a piezoelectric ceramic PZT26 (FerropermMeggit $^{\mathrm{TM}}$, disk ceramic, $2 \mathrm{~mm}$ thick, $15 \mathrm{~mm}$ wide, nominal resonant frequency $1 \mathrm{MHz}$ ) with a water soluble glue. The top of the cavity is closed with a $h_{r e f}=1.1 \mathrm{~mm}$ thick quartz disc (High Tech Materials ${ }^{\mathrm{TM}}$ ), which is both a good acoustic reflector and a good optical transmitter, allowing good optical access for microscopy observations inside the cavity. The position of the quartz lid is guaranteed against surface tension by another ring of aluminium, that is held flat against the quartz with two elastic bands (see Fig. 2 (d) for a global view of the device).

The PZT is powered by a all-in-one wave generator and oscilloscope combo ( $\mathrm{TiePie}^{\mathrm{TM}}$ Handyscope HS5) allowing the variation of the applied voltage between a few $\mathrm{mV}$ to more than $10 \mathrm{~V}$ peak to peak.

It is often more convenient to speak of the acoustic energy density carried by the acoustic standing field in order to compare rigorously different experiments. A calibration is made in our cavity to link $\left\langle E_{a c}\right\rangle$, measured by the method proposed by ${ }^{31}$, with the amplitude of the electric voltage applied to the PZT. The principle consists in measuring the axial focusing velocity of a known particle for a given voltage to deduce the acoustic force, yielding a value for the acoustic energy. 


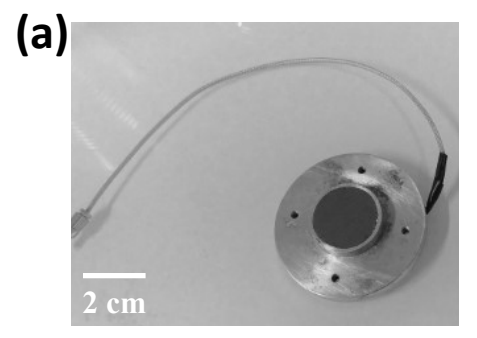

(c)

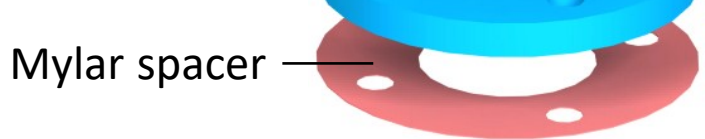

(b)

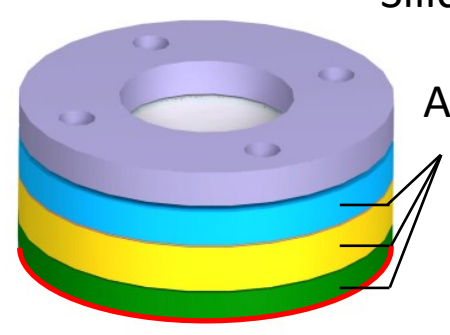

Silicium wafer

Aluminum

parts

Heating

mat (d)

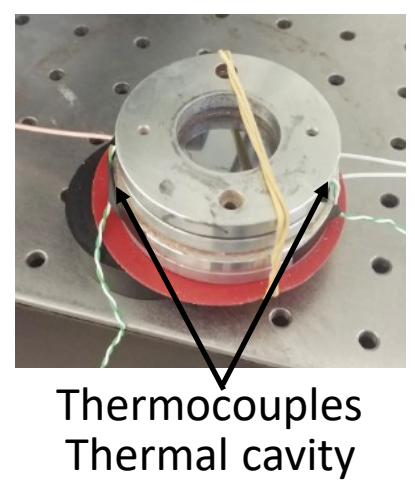

(e)

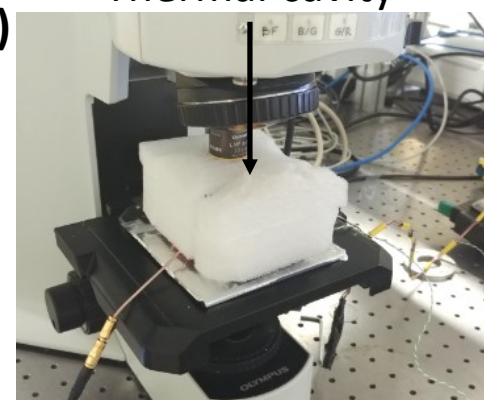

FIG. 2. (a) Picture of the aluminium cavity. (b) Assembled and (c) exploded views of the setup. The aluminium body is made of three aluminium parts: one (yellow) holding a silicon wafer on top of it, another (blue) fitting it and constituting the walls of the cavity. The relative height of resonant cavity is controlled by the insertion of one or several Mylar layers in-between. A piezoelectric transducer (PZT) is glued under the silicon wafer, soldered to a BNC cable. A (green) bottom part closes the device while allowing for cable fitting. A heating mat is stuck under this last part for thermal experiments. The whole device is tightened with screws, and the cavity is closed with a quartz lid held on top of it by an optional second aluminium ring (light grey). (d) View of the thermal acoustic resonator and (e) of the resonator inserted into the insulated chamber fixed on the moving stage of the microscope. 
JASA/Influence of the temperature on the opto-acoustophoretic effect

\section{B. Thermal setup}

In order to control the temperature of the system, we add to our device two elements: a heat source and a thermal isolation. The heat source is a disc-shaped heating silicon mattress (RadioSpares Pro, 4W output power), which is fixed under the aluminium structure. It is connected to a continuous current power source, with a controllable output (RadioSpares Pro). The acoustic cavity has to be isolated from the laboratory air convection, but also to minimize the heat loss. To keep an optical access to the cavity from the top, the aluminium cavity has been inserted inside a carved box made of polyester foam, covered on the inside by a layer of aluminium paper, to keep in also the radiated heat from the device (Fig. 2e).

The aluminium structure temperature could then be monitored using two thermocouples (K type thermocouples connected to a Hanna Instruments HI92804C logging box) that were stuck on the quartz lid sides, where it is easier to get a measurement. We also used a realtime Arduino-based logging device with a generic TMP36GT9Z thermosensor, held down on the silicon wafer center for calibration measurements. The data have been used to measure the dynamic thermal evolution of the cavity, as well as calibrating the slight gap between the two thermocouples placed outside of the cavity and its center.

Several time-evolutions of the silicon wafer temperature were recorded for different thermal powers of the heating mattress and fitted with a simple exponential:

$$
T(t)=\alpha \times\left(1-e^{-t / \tau}\right)
$$


(a)

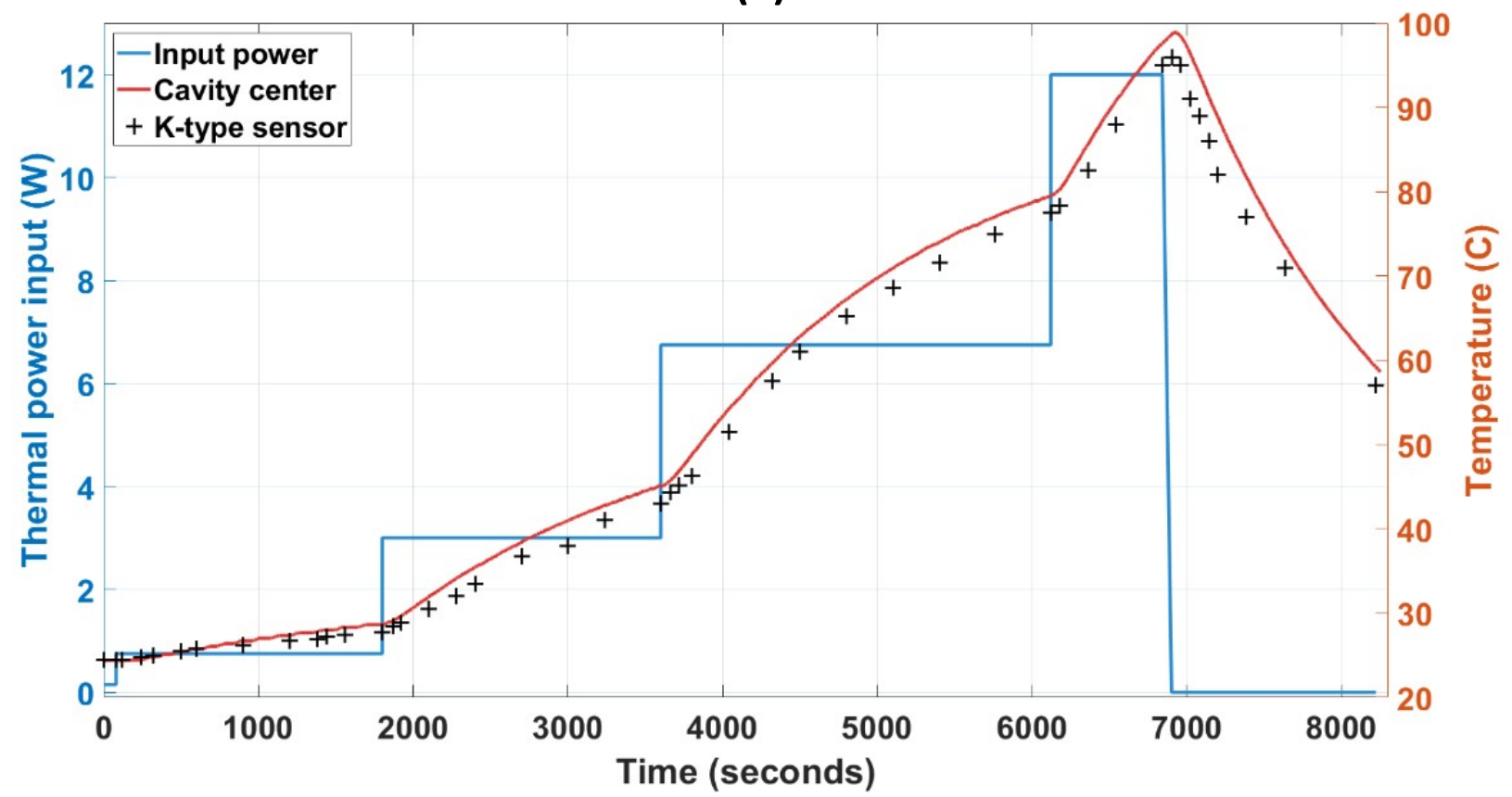

(b)

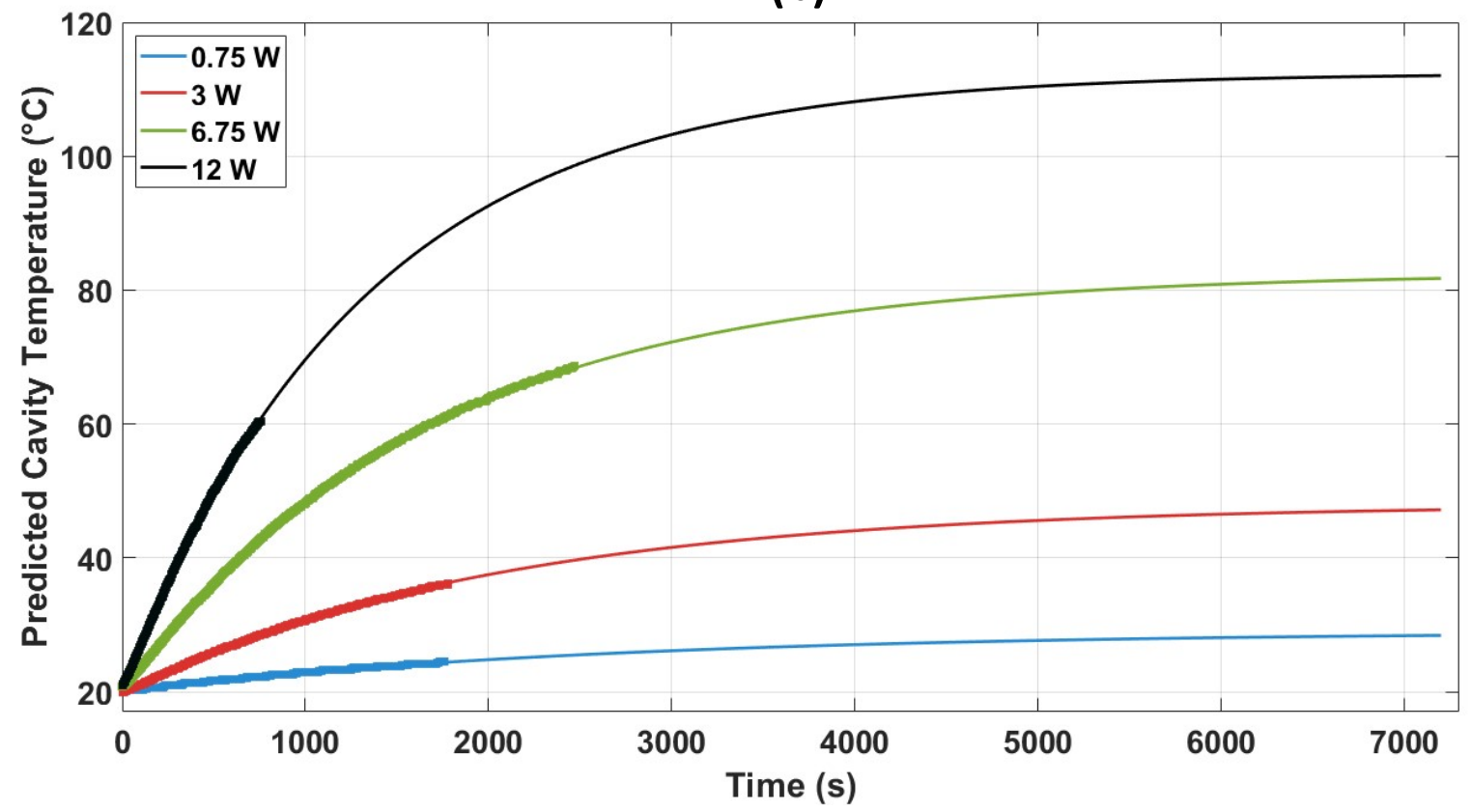

FIG. 3. a) Evolution of the temperature at the center of the silicone wafer as a function of time, for increasing heating powers. b) The temperature evolution for different powers are used to calibrate the thermal response of the setup. Each curve can be fitted with an exponential. Once the dynamic thermal response as a function of the input power is calibrated, it is possible to predict the temporal response of the cavity with the imposed power. 
Once the evolution of $\alpha$ and $\beta=1 / \tau$ with the thermal power is known, it is possible to use it as a calibration to predict the cavity temperature as a function of the applied thermal power (Fig. 3b).

We can also compare the real-time evolution of the thermosensor output with discrete measurements obtained with the thermocouple. As we can see on Fig. 3a, the thermosensor measures a slightly higher temperature, which can be explained by an inhomogeneity of the heat distribution as well as the difference in thermal conductivity paths leading to the two measurement points.

\section{Calibration of the acoustic properties of the heated cavity}

Heating the fluid has various consequences, especially for the acoustics, which have to be taken into account. Heating the fluid changes its density and compressibility, which implies a change of the acoustic contrast factor (eq. 2). However, even for an unrealistic $\Delta T$ of 80 $\mathrm{K}, F_{Y}$ undergoes a relative variation approximating $10^{-5}$, that we will neglect.

The second effect is a modification of the sound velocity in addition to a dilatation of the cavity, affecting the acoustic resonance. For each temperature increase, the new acoustic resonant frequency has been measured by looking for the reflected electric signal minimum, which is a signature of the cavity electromechanical resonance. This way we aim to avoid any errors due to an uncontrolled frequency drift induced by the temperature. Resonant frequency shifted during the experiment from $735 \mathrm{kHz}$ at $20^{\circ} \mathrm{C}$ to $772 \mathrm{kHz}$ at $70^{\circ} \mathrm{C}$, a relative variation of $4.7 \%$, correction which is not negligible and the resonant cavity detuning is then avoided by adjusting the frequency applied to the PZT. 
JASA/Influence of the temperature on the opto-acoustophoretic effect

\section{Optical setup}

The observations are made through the top quartz lid, along the axis of propagation of the acoustic waves, using a reflection microscope (Olympus BX-2 $2^{\mathrm{TM}}$ ), as shown on Fig. 2e.

The samples are illuminated by a LED source (CoolLED pE-4000 ${ }^{\mathrm{TM}}$ ) allowing a very good control of the optical wavelength $\lambda_{\text {opt }}$ (16 different wavelengths between $360 \mathrm{~nm}$ and 800 $\mathrm{nm}$ ) and power of illumination. A $80 \%$ reflective optical filter has been added to the filter cube. Most of the observations were made using a PCO Panda 4.2bi camera at $40 \mathrm{fps}$, when not indicated otherwise. The quantitative measurements were made using a PCO Dimax $\mathrm{CS}^{\mathrm{TM}}$ fast camera at $100 \mathrm{fps}$, to allow a better tracking of the particles. Snapshots were post-treated (contrast improvement, definition of the ROI) with the free software ImageJ (https://imagej.nih.gov/ij/). The power delivered by the CoolLED to the resonant cavity has been measured at the focus of the microscope objectives using a ThorLabs PM16130 Probe (Spectral range 400 to $1100 \mathrm{~nm}, 1 \mathrm{~cm} \times 1 \mathrm{~cm}$ silicon sensor area, optical power range $5 \mathrm{pW}-500 \mathrm{~mW}$ ) connected to a computer.

\section{E. Measurements of the ejection velocity}

In a previous study ${ }^{1}$, the optoacoustofluidics effect was quantified through the slope of a time projection of the aggregate expansion. It gives a good estimate of the global effect of the sudden illumination of an aggregate through its outer boundary ejection velocity.

In this study we improved our measurement technique by using a particle tracking algorithm to measure the velocity of ejected particles. This algorithm was initially developed by 
(a)

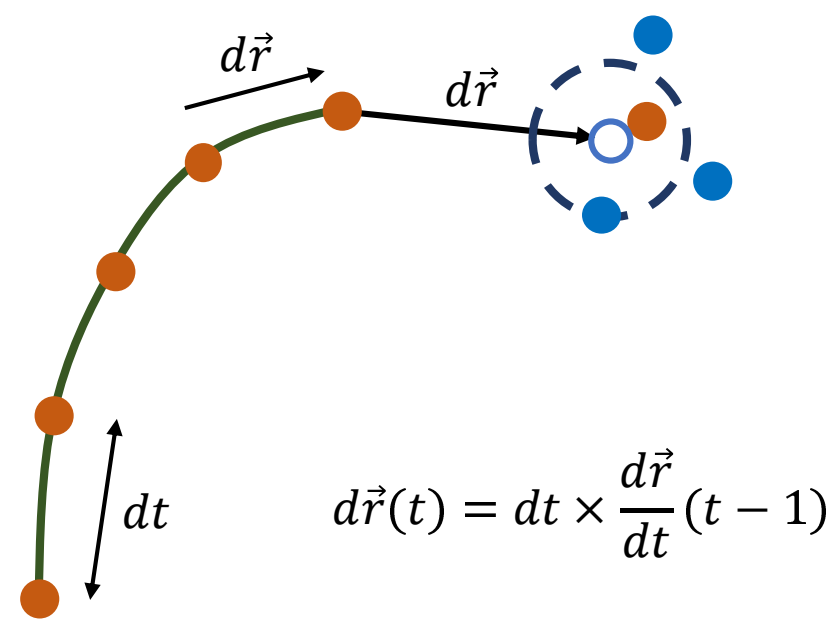

(b)

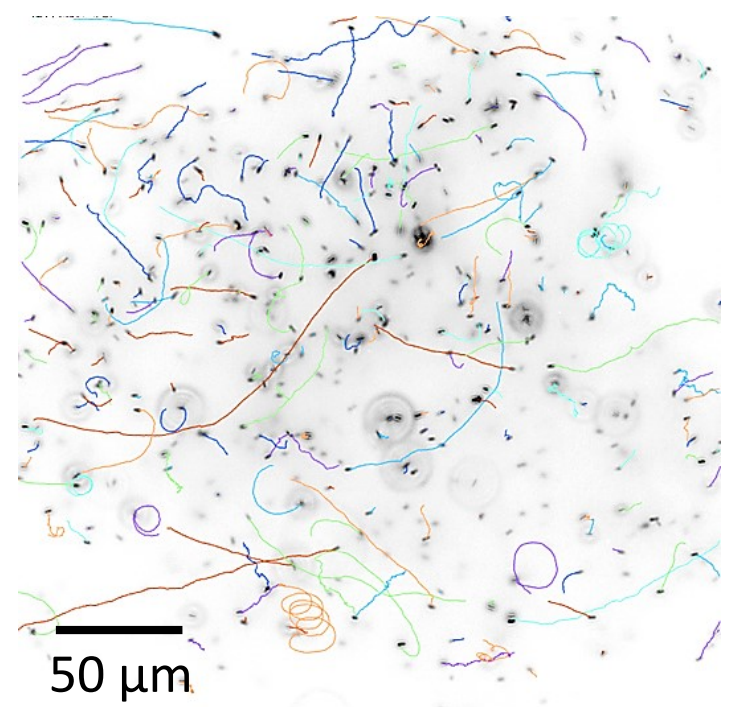

FIG. 4. (a) Sketch of the particle tracking algorithm. A particle is tracked along its trajectory.

the Environmental Complexity Lab, Stanford University, USA ${ }^{32,33}$. The available algorithms have been parallelized and tailored to fit our snapshots time-series.

At the time step $t$, all the local particles have been spotted. To reconnect the "correct" one to the track we are following, we compute the estimated $d \vec{r}$ the particle should have moved during $d t$. Then the closest particle to this guessed position found in the following snapshot $(t+d t)$ is
affected to the track. (b) Example of the simultaneous tracking of a collection of pure Au nanorods
propelled by the ARF and moving with ballistic trajectories inside the levitation plane $(1.84 \mathrm{MHz}$,
$\left\langle E_{a c}\right\rangle \simeq 70 \mathrm{~J} . \mathrm{m}^{-3}$, velocities around $\left.100 \mu \mathrm{m} . \mathrm{s}^{-1}\right)$. $d t$. Then the closest particle to this guessed position found in the following snapshot $(t+d t)$ is
affected to the track. (b) Example of the simultaneous tracking of a collection of pure Au nanorods
propelled by the ARF and moving with ballistic trajectories inside the levitation plane $(1.84 \mathrm{MHz}$,
$\left\langle E_{a c}\right\rangle \simeq 70 \mathrm{~J} . \mathrm{m}^{-3}$, velocities around $\left.100 \mu \mathrm{m} . \mathrm{s}^{-1}\right)$. $d t$. Then the closest particle to this guessed position found in the following snapshot $(t+d t)$ is
affected to the track. (b) Example of the simultaneous tracking of a collection of pure Au nanorods
propelled by the ARF and moving with ballistic trajectories inside the levitation plane $(1.84 \mathrm{MHz}$,
$\left\langle E_{a c}\right\rangle \simeq 70 \mathrm{~J} . \mathrm{m}^{-3}$, velocities around $\left.100 \mu \mathrm{m} . \mathrm{s}^{-1}\right)$. $d t$. Then the closest particle to this guessed position found in the following snapshot $(t+d t)$ is
affected to the track. (b) Example of the simultaneous tracking of a collection of pure Au nanorods
propelled by the ARF and moving with ballistic trajectories inside the levitation plane $(1.84 \mathrm{MHz}$,
$\left\langle E_{a c}\right\rangle \simeq 70 \mathrm{~J} . \mathrm{m}^{-3}$, velocities around $\left.100 \mu \mathrm{m} . \mathrm{s}^{-1}\right)$.

The method consists in a two-step iterative process: (1)

1. All the particles in all the data frames are spotted, according to a threshold's parameters set by the user,

2. Tracks themselves are built by connecting particle positions from frame to frame. 
To allocate a particle to a given track $T_{n}$ at $t+d t$, the routine establishes a guess for the most probable position for the particle at $t+d t$ based on its history at $t, t-d t$, and so on. Then it links to the track $T_{n}$ the particle that minimizes the most the cost function defined as the squared distance between the particle position and this most probable position, as sketched on Fig. 4 a).

The frame rate of the camera is high enough to follow every change in direction of the moving particles. Once the acoustic force is activated, the Brownian motion becomes usually negligible for our particles (larger than $1 \mu \mathrm{m}$ ) leading to a robust prediction of their positions in most of the cases.

Once the particle tracks are recorded from an image sequence of a movie file, they are post-processed according to their quality: noisy data will be filtered by applying a moving average over the positions (generally using a 3 to 5 time-steps averaging), a critical length will be defined for each experiment under which the tracks will be considered as irrelevant, and outliers/parasitic data will be removed from the set.

\section{OPTOACOUSTOPHORESIS}

The phenomenon of acoustic trapping is well understood theoretically for spheres, and its various experimental applications have been extensively covered. Recently, we have shown that if an aggregate of spherical particles in acoustic levitation was illuminated with a proper optical wavelength $\lambda_{\text {opt }}$ with enough power, then the particles are quickly ejected from the illuminated area, while still in acoustic levitation. Indeed, the particles exit the aggregation area radially, while staying focused when observed from the top (see Fig. 5, b and c), meaning 

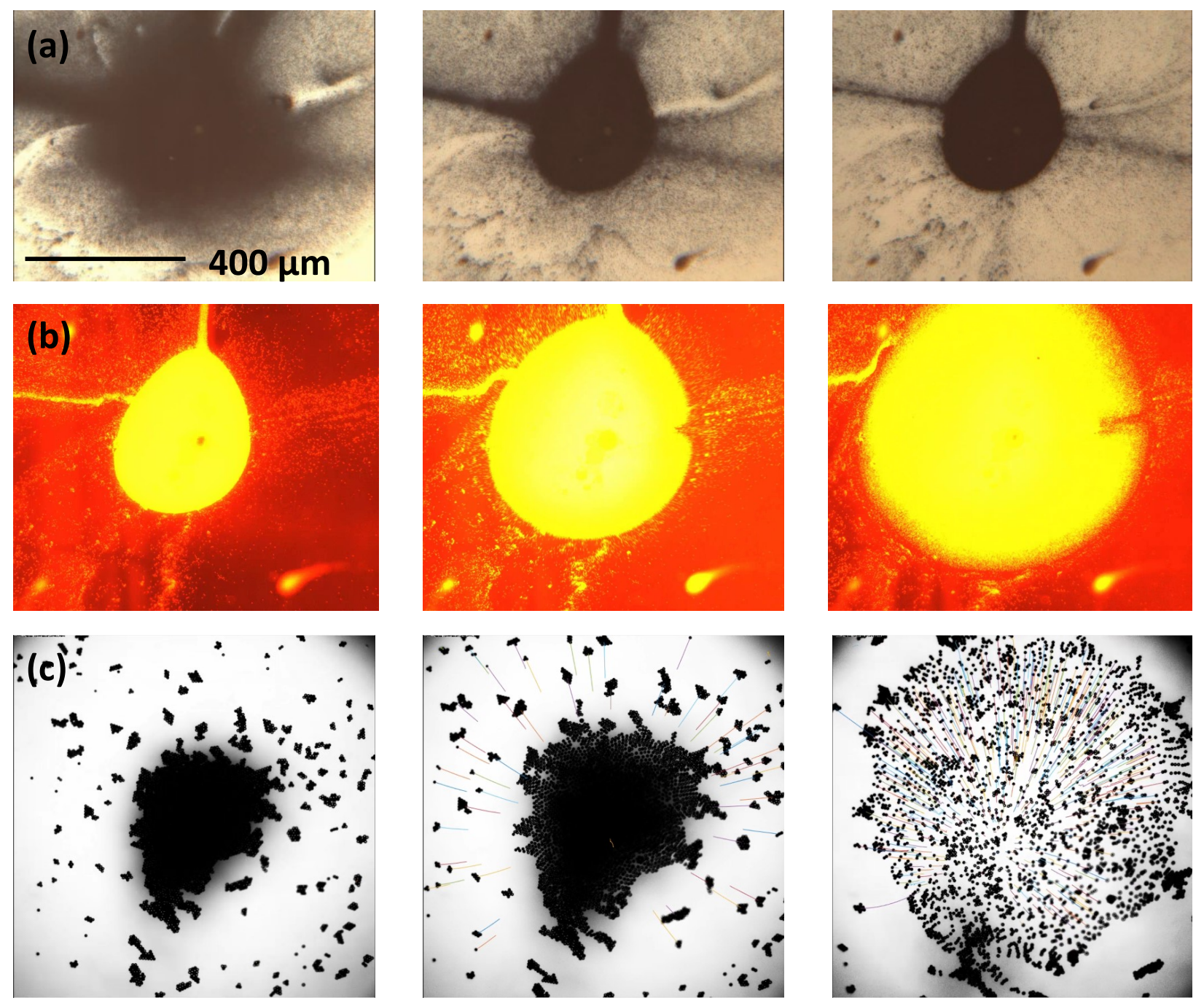

FIG. 5. (a) Illustration of a standard radial aggregation of particles in the acoustic pressure node,

for $1.62 \mu \mathrm{m}$ polystyrene particles in a $1.82 \mathrm{MHz}$ acoustic field. A large aggregate is formed and can be maintained as long as needed in acoustic levitation. (b) If the aggregate is illuminated by a strong green $\left(\lambda_{\text {opt }}=545 \mathrm{~nm}\right)$ light, then the red fluorescent particles, absorbing the green light, are violently ejected from the illuminated area. (c) This "opto-acoustofluidic" effect has also been observed for colored particles (here $10 \mu \mathrm{m}$ red colored polystyrene particles), demonstrating that the fluorescence is not needed. Only light absorption is necessary (maximum effect at $\lambda_{\text {opt }}=550$ or $580 \mathrm{~nm})$. We show particles trajectories superimposed to the snapshots. 
JASA/Influence of the temperature on the opto-acoustophoretic effect

that the $z$ displacement is less than the objective depth of field, i.e. $5 \mu \mathrm{m}$. They are ejected toward the illuminated area frontier where they can aggregate again. The ejection velocities can be as high as few hundreds times their diameter per second.

We illustrate on Fig. 5 c) the disaggregation of a large aggregate of particles at different time steps, along with tracked trajectories measured using the algorithm described in the previous section.

The fact that particles are not ejected isotropically, but stay confined into the levitation plane, ruled out one origin for the phenomenon, i.e. a sign inversion of the acoustic contrast (Eq. 2). Indeed, if the illuminated particles were heating enough to change their material properties compared to the carrying fluid, then the acoustic contrast factor would change sign as well as all the acoustic force components. In this case we should also observe an axial expulsion from the aggregate, with the particles effectively fleeing away from the pressure node isotropically, which is not the case.

A very interesting result was found through a parametric evolution. Indeed it can only be observed with a strong coupling between the optical power and the acoustic energy carried by the acoustic field. The more optical power was used to illuminate the aggregate the stronger the desegregation, which could be expected. But the stronger the acoustic trap, the higher the ejection velocity, which is quite counter-intuitive. This result was reproduced with several sizes of particles, with the same effect for each experiment. It also ruled out a number of possible physical origins for the phenomenon. 


\section{EXPERIMENTAL INVESTIGATION OF A THERMAL EFFECT}

It has been shown recently that fluid layers with a relatively small contrast factor can undergo ARF effects ${ }^{30}$. This is a side effect that can be a strong limitation to the separation of particles transported in layers of fluid of different densities. One could also expect that if the temperature of a particle $T_{\text {part }}$ is increased and then becomes different from the surrounding fluid, then a thermal boundary layer $\delta_{\text {therm }}$ should be created around the particle, or the aggregate of particles (Fig. 6). Increasing the temperature of the fluid leads to a decrease of its density. It is then possible that the particles or aggregates of particles are surrounded by a fluid layer with a density $\rho_{f}\left(T_{1}\right)$ lower than the one of the surrounding fluid $\rho_{f}\left(T_{0}\right)$. Following the proposition of ${ }^{30}$, if two fluids of different densities are subjected to an acoustic field of energy $E_{a c}$, then the fluids will undergo a volume force $F_{a c-f l u}$ defined as:

$$
\boldsymbol{F}_{a c-f l u}=-\cos (2 k y) \cdot E_{a c} \boldsymbol{\nabla} \tilde{\rho}_{f}
$$

where $\tilde{\rho}_{f}$ is the perturbation in fluid density. One can see that if there is a fluid density gradient, then the fluid layer will undergo an acoustic force aligned with density gradients, leading to a possible motion of the fluid layer. The lower density fluid shell surrounding the particle may drag the particle radially, while the particle itself is still kept in acoustic levitation by the primary axial acoustic force. The force applied to the fluid and corresponding drag should be larger than the transverse component of the ARF applied to the particle to pull the particle away from the aggregation site. The resulting ejection speed would then be 
JASA/Influence of the temperature on the opto-acoustophoretic effect

a competition between the transverse component of the acoustic force applied to the particle and viscous drag imposed by the fluid layer moved by $F_{a c-f l u}$.

\section{A. Acoustic instability of the thermal boundary layer}

The objective of these experiments is to assess the role played by possible thermal effects on the opto-acoustofluidic effect. We will rule out a simple thermal convection effect in Section V. One other hypothesis discussed in the previous study was based on successive possible steps triggered when the particles are illuminated. We are interested in the thermal heating of local fluid layers which will undergo a possible acoustic instability.

In any case, if the density gradient induced by local thermal heating is responsible of particle's ejection, then changing the surrounding fluid temperature should modify the ejection process and ultimately, for a high-enough temperature, might cancel it. The objective of following experiments is to check this hypothesis.

\section{B. Influence of the temperature on the ejection velocity}

For these experiments we used $10 \mu \mathrm{m}$ red dyed particles in the thermostated device described in Section II A. Particle concentration was on the order of magnitude of $1 \mu \mathrm{\mu g} \cdot \mathrm{mL}^{-1}$, this concentration yielding for our observation conditions a reproducible aggregate with a correct size. A constant acoustic voltage $U=2.6 \mathrm{~V}_{\mathrm{pp}}$ is applied, while the particles are illuminated at the same optical wavelength $\lambda_{\text {opt }}=550 \mathrm{~nm}$ with the maximum power of the CoolLED. For each temperature, the acoustic frequency is tuned at the proper resonance 

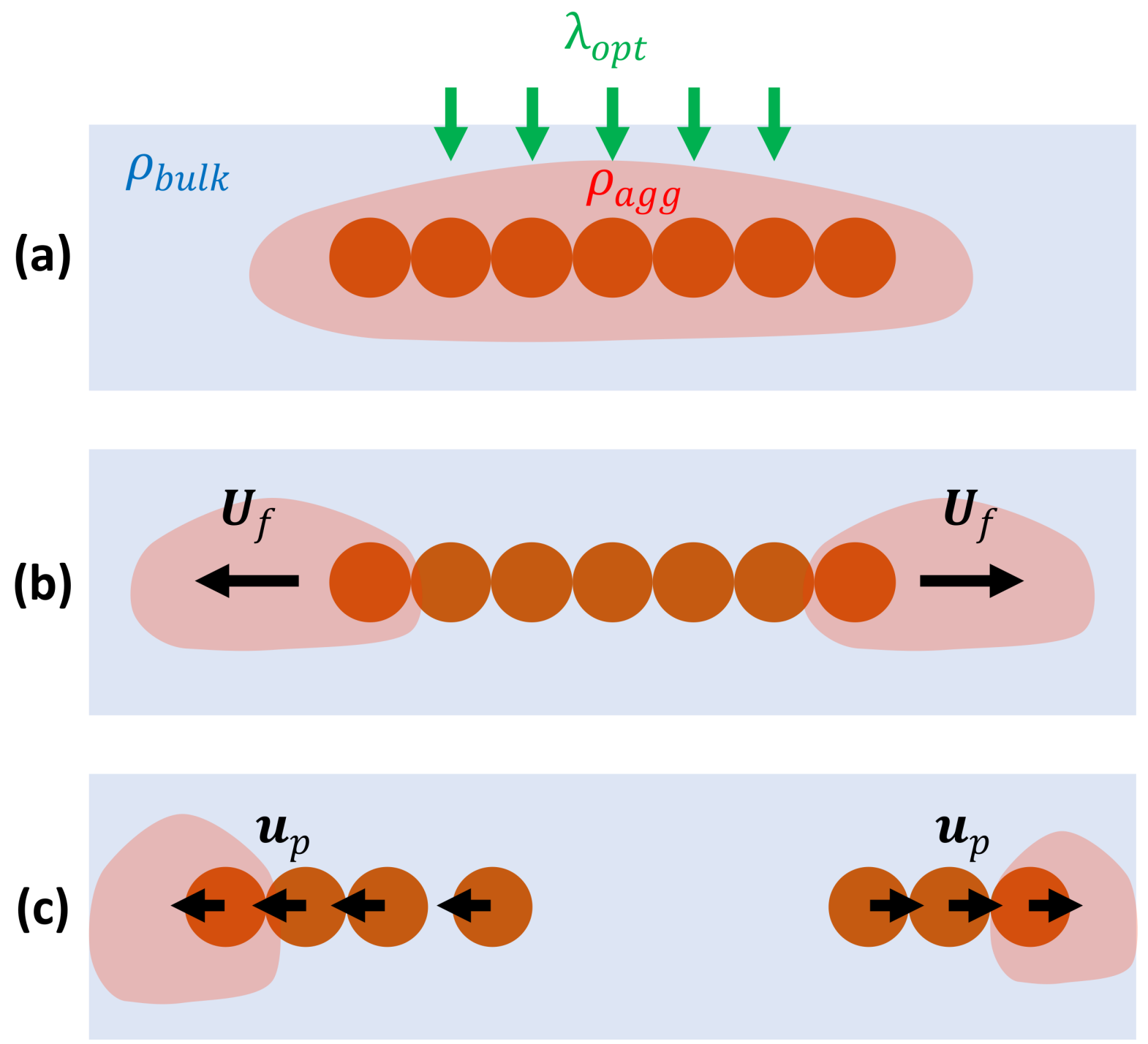

FIG. 6. Illustration of an aggregate who, once illuminated by a specific absorbed wavelength $\lambda_{o p t}$ might absorb the incident energy, therefore heating up the surrounding fluid, thus generating a thermal layer around it (a). The heated layer will then have a lower density than the cooler surrounding fluid, which might generate fluid motion by convection or acoustic instability (b). The particles would then undergo motion through Stokes drag, leading to the observed optoacoustofluidic explosions (c). 


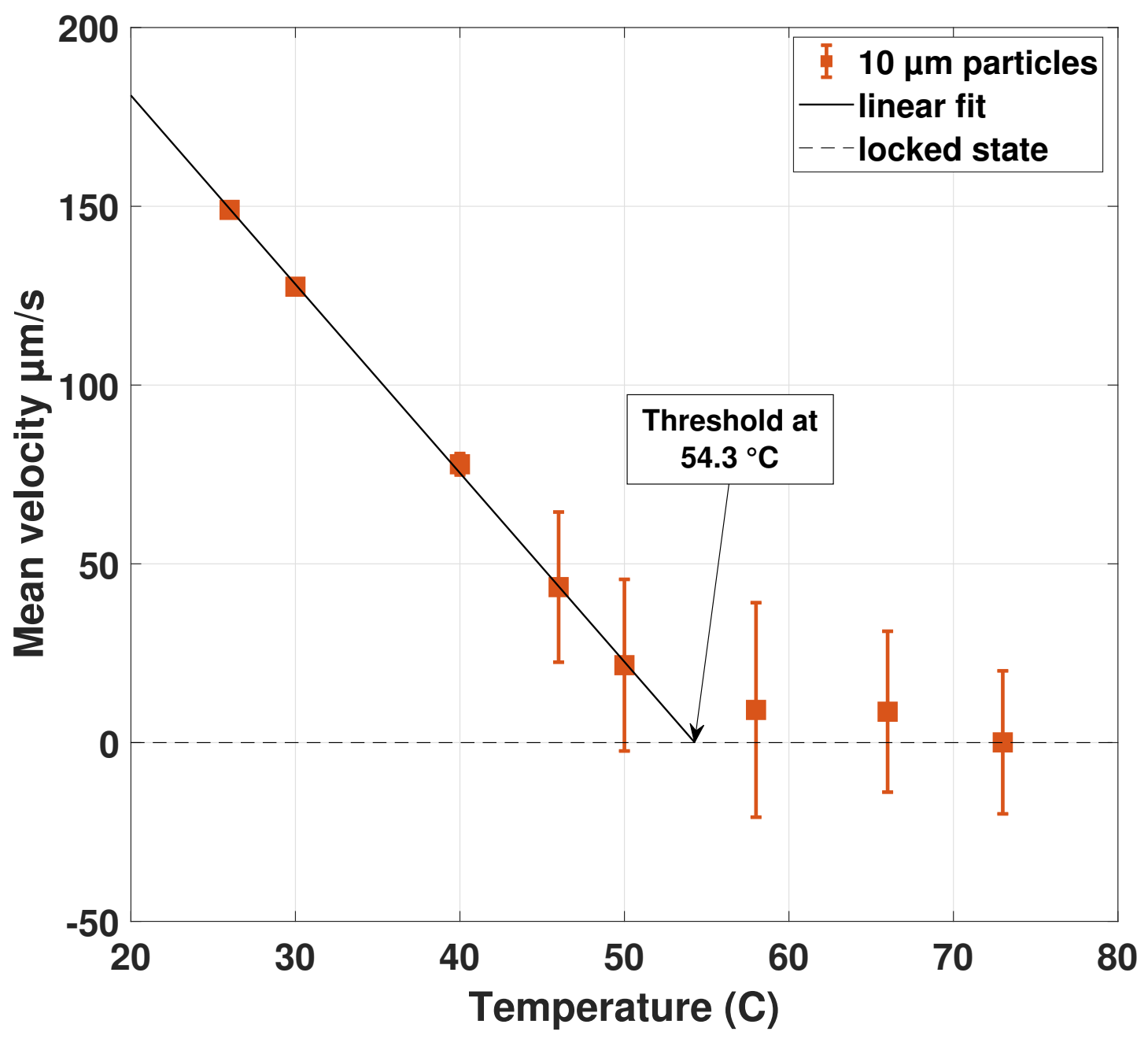

FIG. 7. Ejection velocity of $10 \mu \mathrm{m}$ red dyed polystyrene beads as a function of the temperature. One can see that the ejection velocity is linearly decreasing down to a threshold temperature $T_{\text {crit }}=54.3^{\circ} \mathrm{C}$. For temperatures higher than $T_{\text {crit }}$ the particles remain aggregated in acoustic levitation but no longer react to the illumination. 


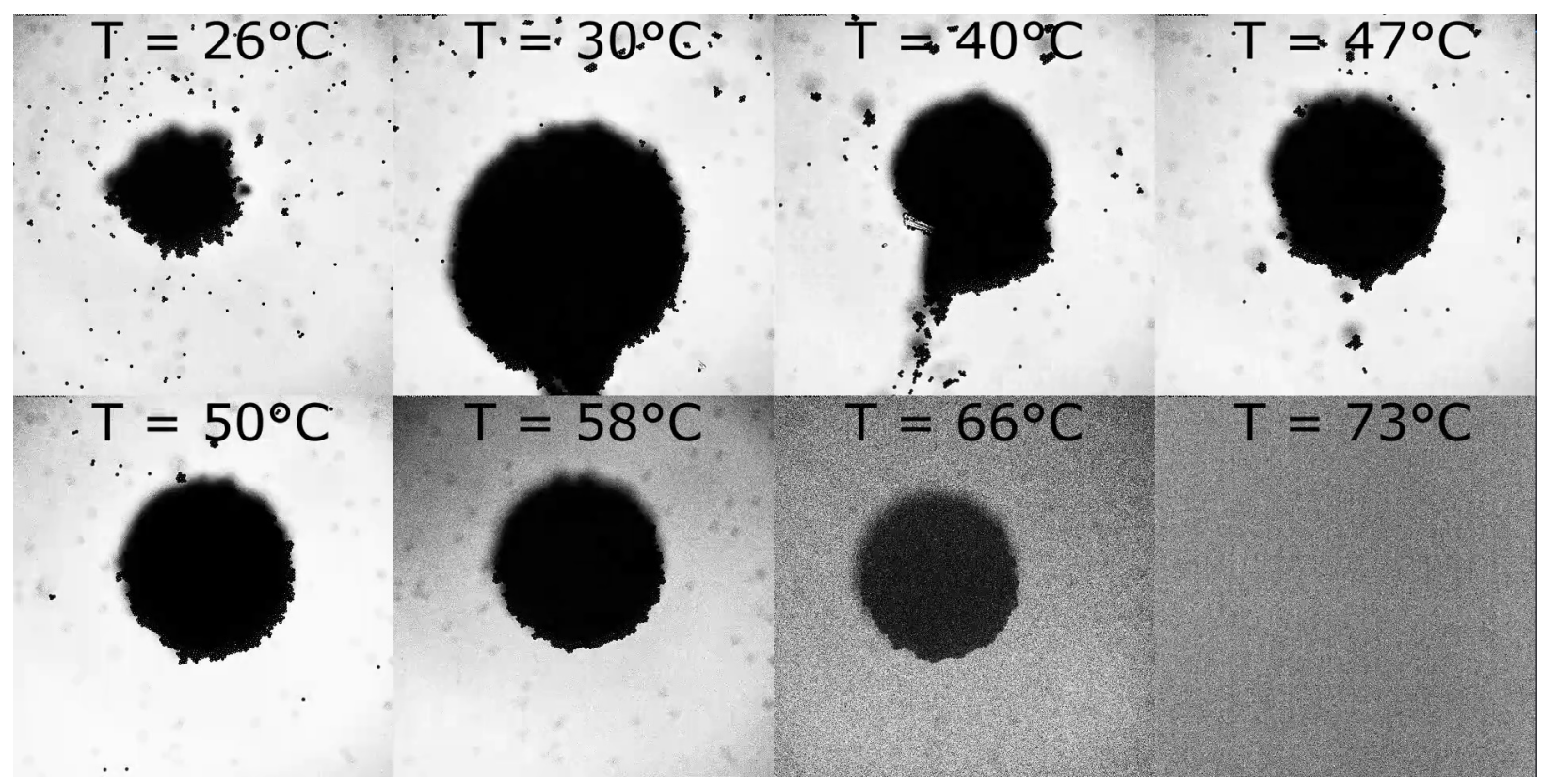

FIG. 8. Illustration of the temperature controlled experiment. $10 \mu \mathrm{m}$ red particles exposed to the same acoustic field and enlightened with the same optical wave are ejected from the aggregation area at different experiment temperatures. One can clearly see the process slowing with the temperature rise.

The trajectories obtained from the Lagrangian tracking of the particles are processed to get their velocity as a function of time, which are then averaged over the number of tracked particles. The results are summarized on Fig. 7. One can notice two interesting features.

1. Ejection velocity decreases linearly with increasing temperature

2. There exists a critical temperature above which the particles are no longer ejected from the illuminated area, in this case $54.3^{\circ} \mathrm{C}$. 
It is important to note that since these experiments are also conducted at slightly different frequencies thanks to the cavity heating (as described in II C). When conducting control experiments at these frequencies but with $T=20^{\circ} \mathrm{C}$, the ejection was effective.

This results clearly demonstrate that the temperature difference between the bulk fluid and the heated layer surrounding the particles plays a critical role. Once the bulk fluid becomes hotter than the heated layer, the expulsion mechanism is turned off. This result is compatible with the idea of a heated fluid layer being pushed by the acoustic force. To check this theory, we carried out numerical simulations on simple configurations including the various forces playing a role in this phenomenon.

\section{NUMERICAL INVESTIGATION}

We present in this section numerical simulations that were conducted with Comsol Multiphysics 5.1, in order to evaluate the effects of the heated layer surrounding a particle or an aggregate discussed previously. The simulations are used as a tool to have a better insight into the local effects discussed previously. We will see that small temperature gradients could generate large flows if the acoustic force is taken into account.

The main physical parameters used for the simulations are summarized in Table I.

All the simulations were performed on a DELL Precision 7820 tower computer running Windows 10 Professional for workstations (64 bits), equipped with with 64 GB of RAM and two Intel Xeon Bronze 3106 CPUs with 8 cores each, cadenced at $1.7 \mathrm{GHz}$ and attached to $11 \mathrm{MB}$ of cache memory. 
JASA/Influence of the temperature on the opto-acoustophoretic effect

\section{A. Rayleigh-Bénard instability}

When dealing with thermal effects in fluids, the first question is whether or not the fluid can undergo Rayleigh-Bénard instability. The previous experiment suggest a possible temperature difference $\Delta T \approx 35^{\circ}$ when running an experiments at room temperature $\left(20^{\circ}\right.$ C). In this case one should compute the Rayleigh number, defined as:

$$
R_{a}=\frac{g \rho_{f} \alpha_{w}}{\nu D_{t h}} \times \Delta T \cdot L^{3}=G_{r} \times P_{r}
$$

where $G_{r}$ is the Grashof number and $P_{r}$ is the Prandtl number. $D_{t h}$ is the thermal diffusion coefficient, $L$ the length scale over which the thermal gradient takes place, $\alpha_{w}$ is the thermal expansion coefficient and $\nu$ is the kinematic viscosity.

Using the values of the various physical parameters summarized in Table I, it is possible to compute extremes values of the Rayleigh number as a function of the temperature. Even with unreasonable control parameters $\left(\Delta T=100\right.$ degrees, $\mathrm{L}=1 \mathrm{~mm}$ yield $\left.R_{a} \simeq 10^{3}\right)$ the Rayleigh numbers remains lower than the critical value $\left(R_{a} \leq R_{a}^{\text {crit }}=1708\right)$ above which the thermal convection is triggered. The ejection of the particles when illuminated can not be explained by a simple thermal convection effect.

\section{B. Geometry, boundary conditions and mesh definition}

As we are interested in evaluating the coupling effect of thermal heating and acoustic entrainment, we neglect possible 3D effect and simplify the geometry of the experimental setup. We use the cylindrical symmetry of the problem to model the cylindrical cavity as 
JASA/Influence of the temperature on the opto-acoustophoretic effect

TABLE I. Table summarizing the main physical parameters characterizing the fluid and the polystyrene particles. We used these parameters to compute the temperature dependency of the Rayleigh number as well as for the numerical simulations. We used the values of the COMSOL Material Library as well as their temperature dependence.

Polystyrene

Density

Speed of sound

Compressibility $\rho_{p s}$

$c_{p s}$

$\beta_{p s}$
1060

2350

$2.48 .10^{-10}$ $k g \cdot m^{-1}$

$m . s^{-1}$

$P a^{-1}$

Water

Density $^{\mathrm{a}}$

Speed of sound

Compressibility

Kinematic viscosity

Thermal conductivity

Heat capacity

Thermal expansion coeff.

Thermal diffusivity

Prandtl number ${ }^{\mathrm{b}}$

Grashof number

Rayleigh number ${ }^{\mathrm{d}}$ $\rho_{w}$

$c_{w}$

$\beta_{w}$

$\nu$

$k_{t h}$

$C_{p}$

$\alpha$

$D_{t h}$

$P_{r}$

$G_{r}$

24
999

1481

$4.57 .10^{-10}$

$1 \times 10^{-6}$

0.6

4180

$J . k g^{-1} \cdot K^{-1}$

$0.17 \times 10^{-3}$

$1.43 \times 10^{-7}$

$m^{2} \cdot s^{-1}$

$m . s^{-1}$

$P a^{-1}$

Pa.s

$W \cdot m^{-1} \cdot K^{-1}$

$K^{-1}$ $\mathrm{kg} \cdot \mathrm{m}^{-1}$

7.5

8.5

$R_{a}$

76 
JASA/Influence of the temperature on the opto-acoustophoretic effect

a rectangular domain (cross-section of the cavity). The side walls are made of aluminium, while the bottom wall is made of silicon and the upper wall is made of quartz. The simplified computational domain is then rectangular with a width $w=4 \mathrm{~mm}$ and height $h=400 \mu \mathrm{m}$.

As the PZT used in the experiments has a smaller diameter than the overall cylindrical cavity, we define an "active area" of width $w_{a c}=1 \mathrm{~mm}$ (Fig. 9), where the acoustic force defined in Eq. 5 is active.

We do not simulate acoustic waves emission and reflections in these simulations. The only acoustic effect is computed as a body force. All solid boundaries are defined as hard walls with specific thermal properties. A constant temperature is applied to the outer walls of the cavity, the same way as in the experimental device.

We model the aggregate as a set of $2 \mu \mathrm{m}$ diameter beads stuck with each other in the geometrical center of the resonator (Fig. 9 c)). The position of the aggregate is fixed to represent the initial condition of a stable aggregate in acoustic levitation just before the illumination. We don't consider a possible motion of individual particles. The objective is to compute the possible fluid motions only induced by thermal effects and the acoustic field.

We make the hypothesis that the particles absorb the energy injected through the illumination. This illumination is then modelled through a given heat injected by the aggregate into the surrounding fluid. The top surface of the aggregate is defined as a constant heat output flux, and its bottom boundary is thermally insulated. This way we try to take into account the fact that the aggregates are illuminated from the top. Changing this condition to set both the top and bottom boundaries of the particles to be heated did not change the output of the simulation. 


\section{(a)}

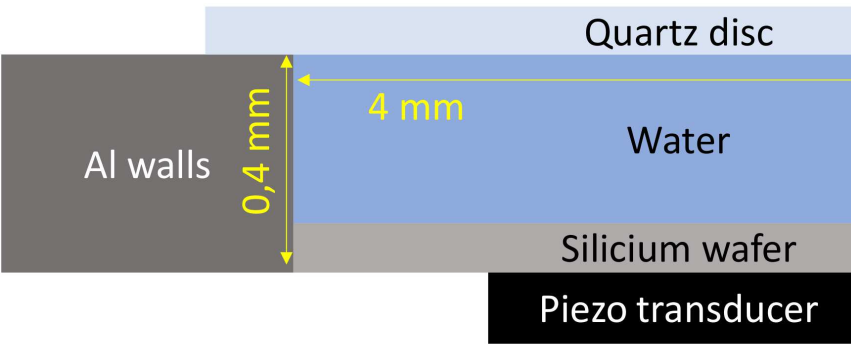

(b)

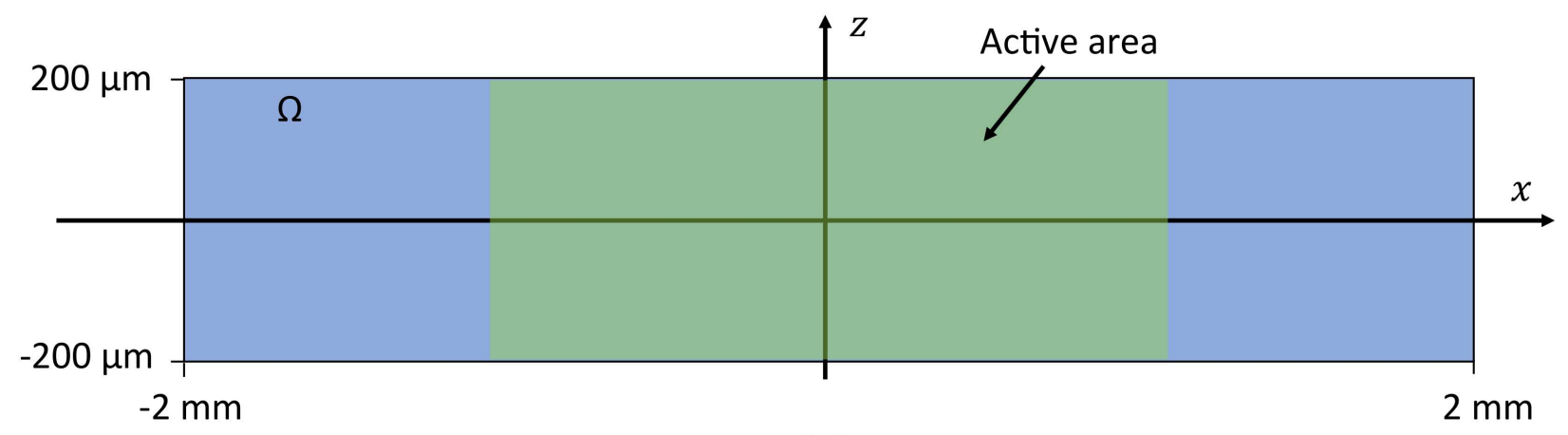

(c)

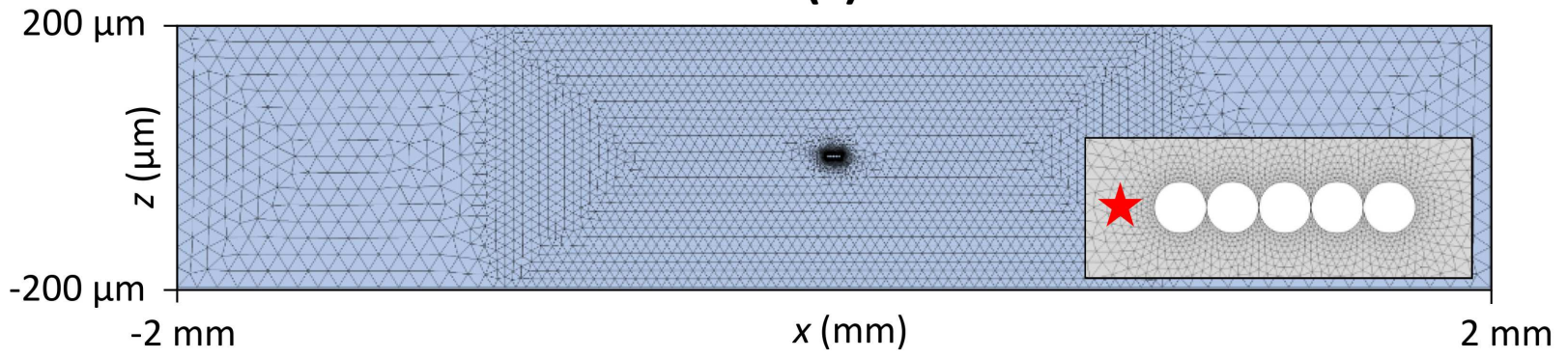

FIG. 9. a) Cross-section of the model acoustophoretic resonator used in his work. It is a cylindrical aluminium cavity, with a silicone wafer as a transmitter layer, on which a PZT element is glued. The reflecting layer is a quartz lid, which is both a good acoustic reflector and a good optical transmitter. b) The corresponding two dimensional computational domain $\Omega$. The fluid cavity is closed by rigid walls with specific thermal properties. To account for the fact that the PZT is smaller that the cavity, a subdomain in which the acoustic force is applied is created. It is called the "active area". (c) The aggregate is modelled as a raft of five $2 \mu \mathrm{m}$ beads fixed in the middle of the cavity, in a similar position as in the acoustic levitation case. On the right part of the figure we added a zoom of the mesh around the bead6, together with a red star indicating the location of a local probe used to monitor the fluid velocity. 
For the initial conditions the heat output of the aggregates to a given value, while the bulk temperature of the resonator is set to the temperature of the outer walls.

To define the proper size of the smallest element of the mesh we evaluate some important length scales: the thermal $\delta_{t h}$ and the viscous $\delta_{v i s c}$ penetration depths, that can be written as:

$$
\delta_{t h}=\sqrt{\frac{2 \gamma D_{t h}}{\omega}} \text { and } \delta_{v i s c}=\sqrt{\frac{2 \mu}{\rho_{f} \omega}}
$$

leading to $\delta_{t h}=0.16 \mu \mathrm{m}$ and $\delta_{v i s c}=0.43 \mu \mathrm{m}$ for a $1.875 \mathrm{MHz}$ acoustic field at $20^{\circ} \mathrm{C}$. We use these length scales to define the size of the smallest element of the mesh used for these simulations.

The mesh is conditioned by two parameters. One controls the maximum element size of the grid in the bulk, several elements from the simulated aggregate, while the other controls the number of elements on the aggregate boundaries, effectively forcing the local density of elements in the aggregate vicinity.

The first parameter, $d_{b u l k}$, controls the precision with which the physical fields in the bulk will be rendered. The second, $N_{\text {part }}$, ensures a good coverage of the two length scales around the aggregate as defined in Eq. 7. $d_{b u l k}$ is best represented as a fraction of the aggregate width, to account for the physics of the problem. $N_{\text {part }}$ is defined as the number of boundary elements surrounding a particle. In this case, we use $2 \mu \mathrm{m}$ particles, leading to an element size of $0.1 \mathrm{\mu m}$ for $N_{\text {part }}=62$. The growing factor of the element size from the boundary to the bulk has been set to 1.1, ensuring that the elements keep the same size over two micrometers, to cover the thermal length scale at least 4 times. 
In the following, a maximum bulk element $d_{b u l k}=0.1 L_{a g g}$ is selected, and a number of boundary elements for the aggregate of $N_{\text {part }}=800$ is kept, resulting in a mesh of $3.1 \times 10^{6}$ elements.

\section{Volume forces}

The body buoyant forces are represented as a volume force, active over the whole domain $\Omega$, defined as:

$$
\boldsymbol{f}_{\text {buoy }}=-\rho_{f} \times g \boldsymbol{e}_{z}
$$

with $g$ the standard gravity. It will take into account any density difference effects, especially the ones induced by local heating.

As mention previously the acoustic force applied on fluids (eq. 5) is taken into account only in the active domain described on Fig. 9.

As we are interested in thermal effects over a relatively large range of temperature we used the temperature dependency for all physical parameters (density, viscosity, thermal conductivity, ...) as defined in the Comsol Material Library.

\section{Natural convection}

We first ran a test simulation on a very classical Rayleigh-Bénard case, where only buoyancy and gravity were acting on the fluid. One can expect to observe natural convection rolls once above the critical Rayleigh number. Nevertheless, we have shown in Section VA that 
$R_{a}^{\text {crit }}$ cannot be reached unless an unphysical $(\Delta T>100)$ very large temperature gradient is applied in a taller cavity than the one used here.

We used a rectangular box, $800 \mu \mathrm{m}$ tall and $400 \mu \mathrm{m}$ wide, with hot bottom plate and a cold top plate. The other wall were insulated. We did not observe any onset of the convective instability, which is coherent with the Rayleigh numbers explored here.

Moreover, $R_{a}^{\text {crit }}$ depends on the lateral confinement of the fluid, i.e. on the aspect ratio of the simulated box as shown by ${ }^{34}$. The critical Rayleigh number $R_{a}^{\text {crit }}$ also scales with lateral confinement of the flow as $(h / d)^{435}$, with $h$ and $d$ the height and width of the cavity. In our case, it leads to a corrected critical Rayleigh number $R_{a}^{\text {crit }} \approx 27000$. It confirms that the results of the simulation are correct and that there should not be any convective effect inside the cavity.

\section{THERMO-ACOUSTOFLUIDIC SIMULATIONS}

\section{A. Thermo-acoustofluidic instability}

In the following, we include in the simulation the acoustic force acting on fluids. The acoustic energy for all the simulations is $\left\langle E_{a c}\right\rangle=10 \mathrm{~J}^{-3}{ }^{-3}$, which is a standard value for our cavities.

We show on Fig. 10a the contour plot of fluid flow magnitude, together with vector plots of the flow created inside the cavity when the upper part of the aggregate is heated. One can clearly see four large re-circulations with a very different organization than the Rayleigh-Bénard instability. Two large horizontal velocity area appears on both sides of 
(a)

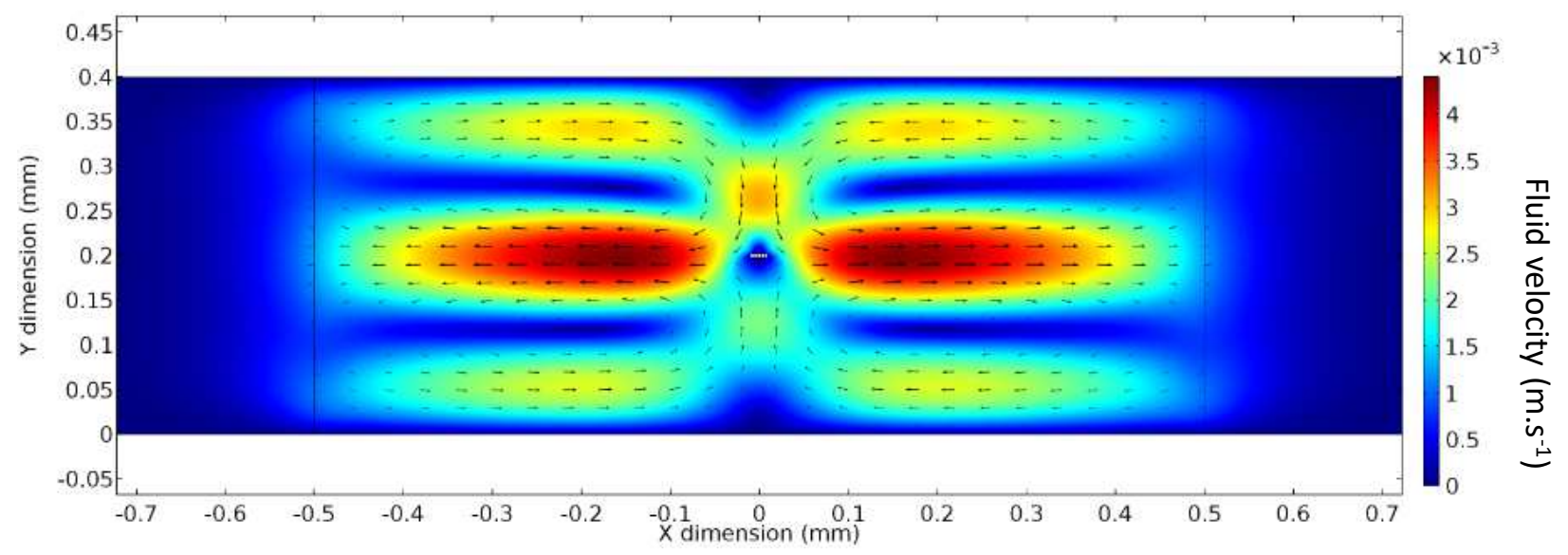

(b)

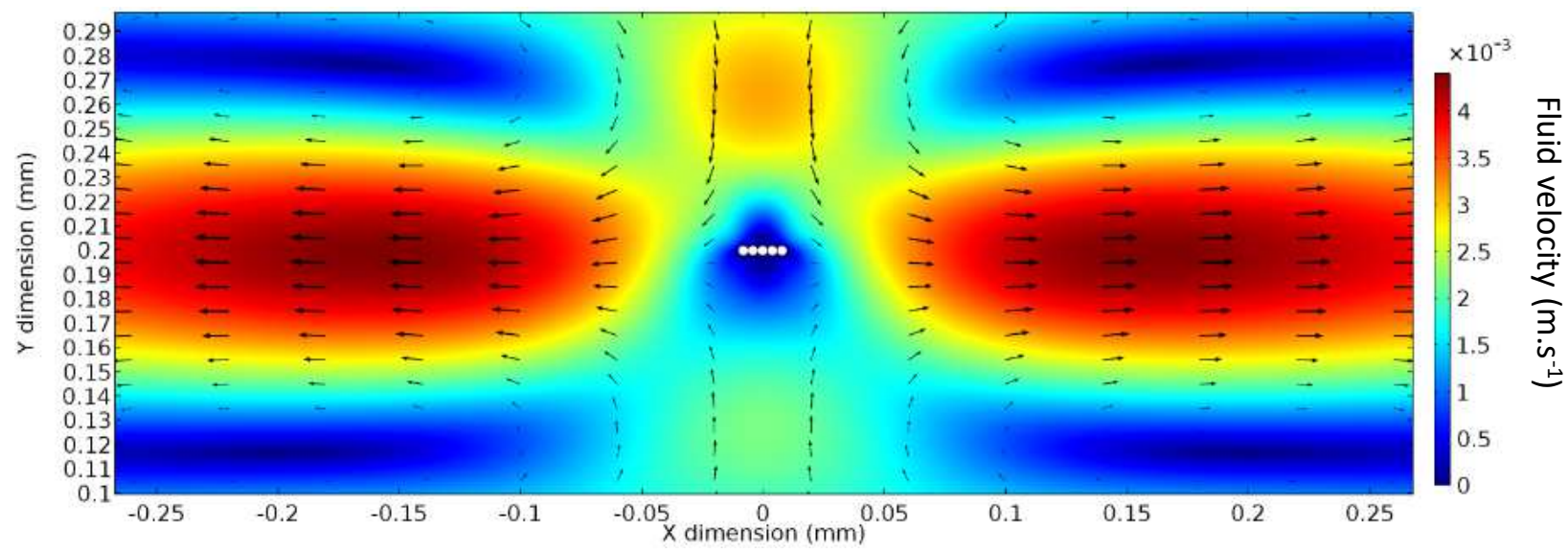

FIG. 10. a) Final steady state of our cavity under the influence of buoyant forces and acoustic forces. The traditional convective recirculation is reversed, and the fluid exits the hot area by the sides, looping back on top and bottom to close the recirculation loops. b) Close-up on the aggregate area, where we can see a slight asymmetry of velocity over and under the aggregate, caused by the fact that this one is rigid. A movie exposing the phenomenon dynamics is available: MM1, this is a file of type "mp4" (2.90Mb). 
the aggregates. The resulting counter-rotating rolls induce a clear down-flow toward the aggregate, in the opposite direction of what would be expected for a convective flow.

This result may look counter-intuitive but it illustrates a very plausible mechanism for the opto-acoustofluidic effect. Indeed, the particles absorb the light before heating a fluid layer, which in turns see its density decrease. Instead of moving up as in a classical RayleighBénard instability, the fluid is entrained radially by the acoustic force toward the outer walls of the cavity. Once the lighter fluid exit the active region, the classic mechanism prevails and the less dense fluid rises toward the upper wall, triggering a large-scale flow inside the cavity. From this point of view, one can consider this phenomenon as new hydrodynamic instability, which could be called "thermo-acoustofludic" instability, as it can trigger the creation of large-scale rotating flows opposite to classic Rayleigh-Bénard instability.

This mechanism is compatible with several key properties of the optically induced particle ejection. It exhibits a fluid motion that can explain the particles ejection from the aggregate periphery. Indeed, in this simulations the aggregate is fixed, so that the heated layer are radially ejected. In the experiment particles are in levitation and can be dragged by the Stokes force induced by this acoustically forced fluid motion. Moreover the fluid motion is centered on the levitation plane, which explains why the particles are radially ejected and kept inside the levitation plane.

\section{B. Scaling of the fluid ejection velocity}

We made a parametric study to evaluate the influence of both the acoustic energy and the thermal heating of the fluid ejection velocity. The fluid velocity is monitored on the 
(a)

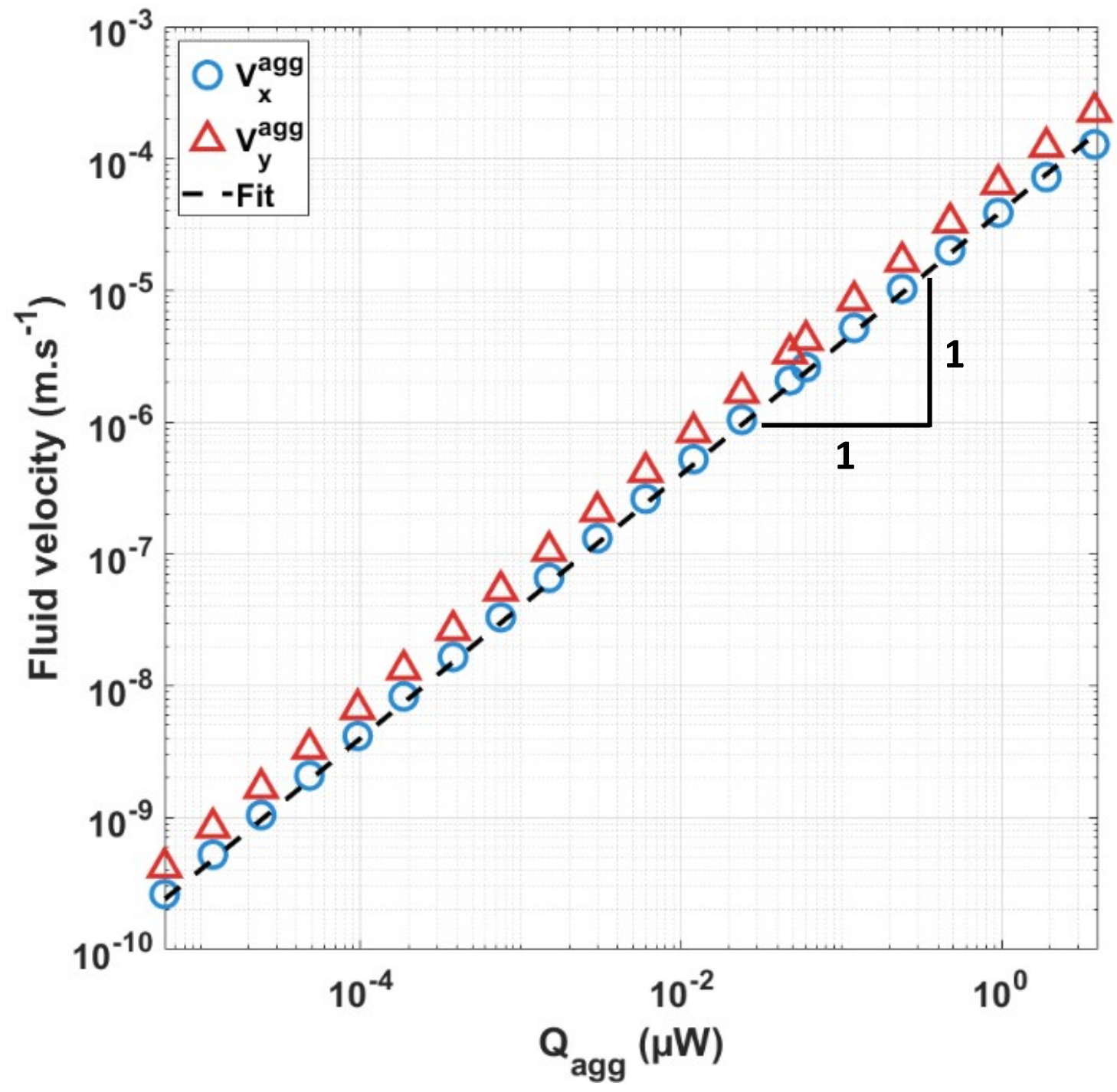

(b)

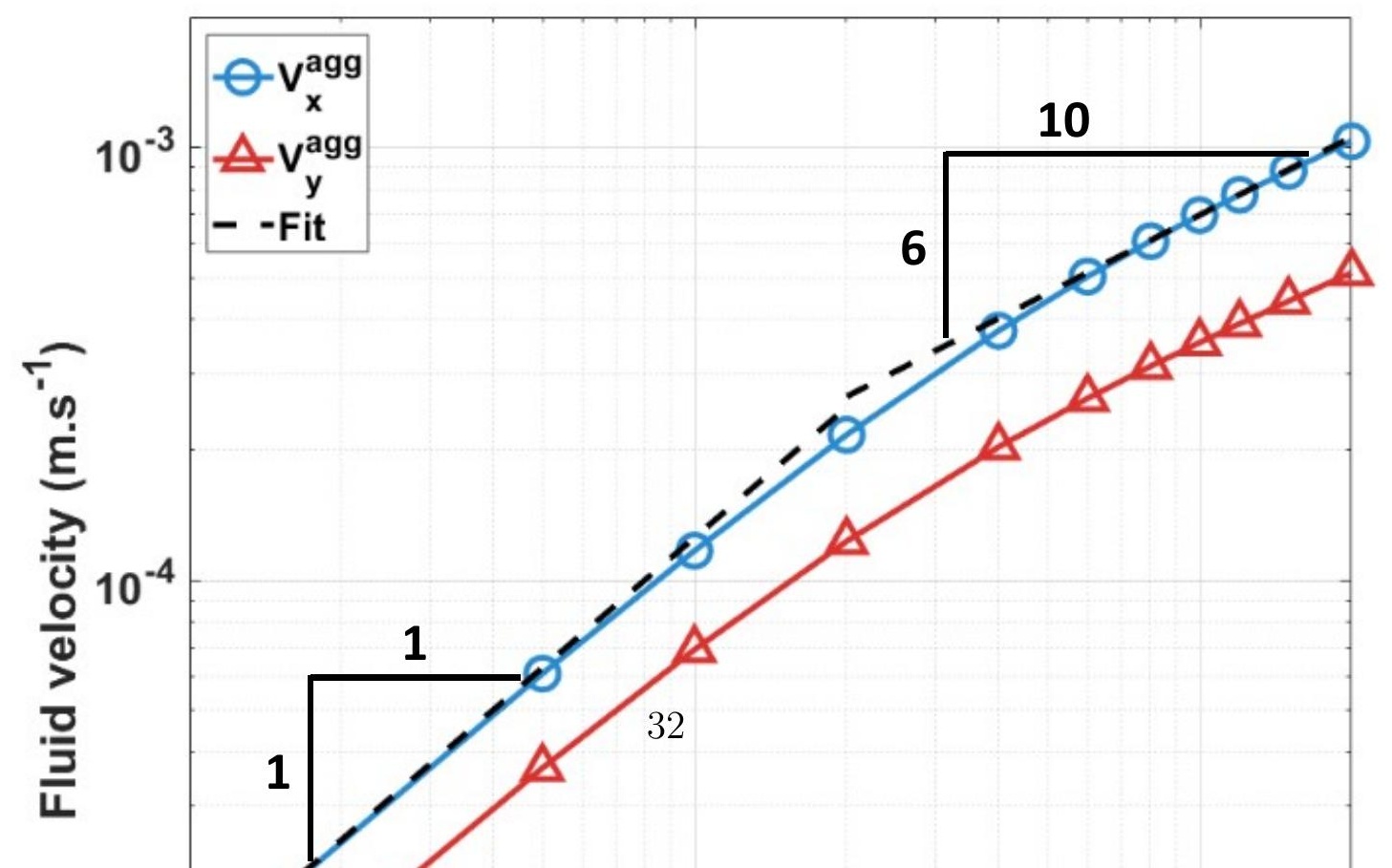


local probe, on the aggregate side, as shown on Fig. 9 (c). The fluid ejection velocity can then be plotted as a function of the heat output (Fig. 11a) and of the acoustic energy (on Fig. 11b).

We find a positive scaling of the fluid velocity when increasing both the illumination power and the acoustic energy of the field, which matches our observations. Even more, the scaling is in good agreement with what we found in experiments with very small particles, which are very prone to follow fluid motion: a linear scaling for increasing illumination power, and a fraction power law with the acoustic energy. Fluid velocity evolution with the acoustic energy increase only has a tangential linear evolution at the origin of this graph, and a $3 / 5$ scaling, close to the $3 / 4$ scaling found in the previous experimental study ${ }^{1}$.

\section{Ejection velocity of particles}

As mentioned previously, the numerical simulations were used to demonstrate that a fluid flow could be triggered if both thermal effect and acoustic force applied on fluids of different densities are taken into account. A possible explanation of the opto-acoutofluidic effect is then the entrainment of particles by the ejected fluid. In this case, we have to take into account two important effects which will make the particle ejection velocity different from the fluid ejection velocity: first, the Stokes drag which will slow the particles and, second, the transverse component of the ARF, which will also slow the particle down.

Balancing the forces applied to the particles lead to the following equation:

$$
\rho_{p} V_{p} \frac{\partial \boldsymbol{u}_{p}}{\partial t}(x, y)=\boldsymbol{F}_{T}+\boldsymbol{F}_{\text {Stokes }}
$$


where $V_{p}$ is the volume of the particle, $F^{r a d}$ the radiation force and $F_{\text {Stokes }}$ the Stokes force applied to the moving particle. It leads to the following equation in the levitation plane:

$$
\begin{array}{r}
\frac{\partial \boldsymbol{u}_{p}}{\partial t}(x, y)+\frac{3 \pi \eta d_{p}}{V_{p} \rho_{p}} \times \boldsymbol{u}_{p}(x, y)= \\
\frac{1}{\rho_{p} V_{p}}\left(\boldsymbol{F}_{T}(x, y)+3 \pi \eta d_{p} \boldsymbol{U}_{f}\right)
\end{array}
$$

where $\boldsymbol{F}_{\text {rad }}^{\text {radial }}$ is the transverse component of the ARF. The solution of a simple first degree differential equation of the kind $y^{\prime}+a y=b$ being of the form $y=-b / a \times\left(e^{-a t}-1\right)$, a general solution for these equations can be:

$$
\begin{aligned}
\boldsymbol{u}_{p}(t, x, y) & =\frac{\boldsymbol{F}_{T}(x, y)+3 \pi \eta d_{p} \boldsymbol{U}_{f}}{3 \pi \eta d_{p}} \\
& \times\left[1-\exp \left(-\frac{3 \pi \eta d_{p}}{V_{p} \rho_{p}} t\right)\right]
\end{aligned}
$$

Using this relation, we can go further and try to build a phase diagram showing how the ejection velocity of the particle depends on the illumination power and acoustic energy. To do so we first have to define a value for the transverse component of the ARF $F_{\text {rad }}^{\text {radial }}$. We set it to $10^{-8} \mathrm{~N}$, which is quite high (usually around $10^{-12} \mathrm{~N}$ in the literature ${ }^{28,29}$ ), meaning that our simulation covered a larger range of optical power than our experiment. Then we used the horizontal fluid velocity obtained through the simulations, right at the aggregate side, and plugged it into Eq. 11 to translate it into particle ejection velocity.

The result of our simulation can be found on Fig. 12, which is a phase diagram showing the dependency of the ejection velocity of the particles to both the acoustic energy and 
(a)
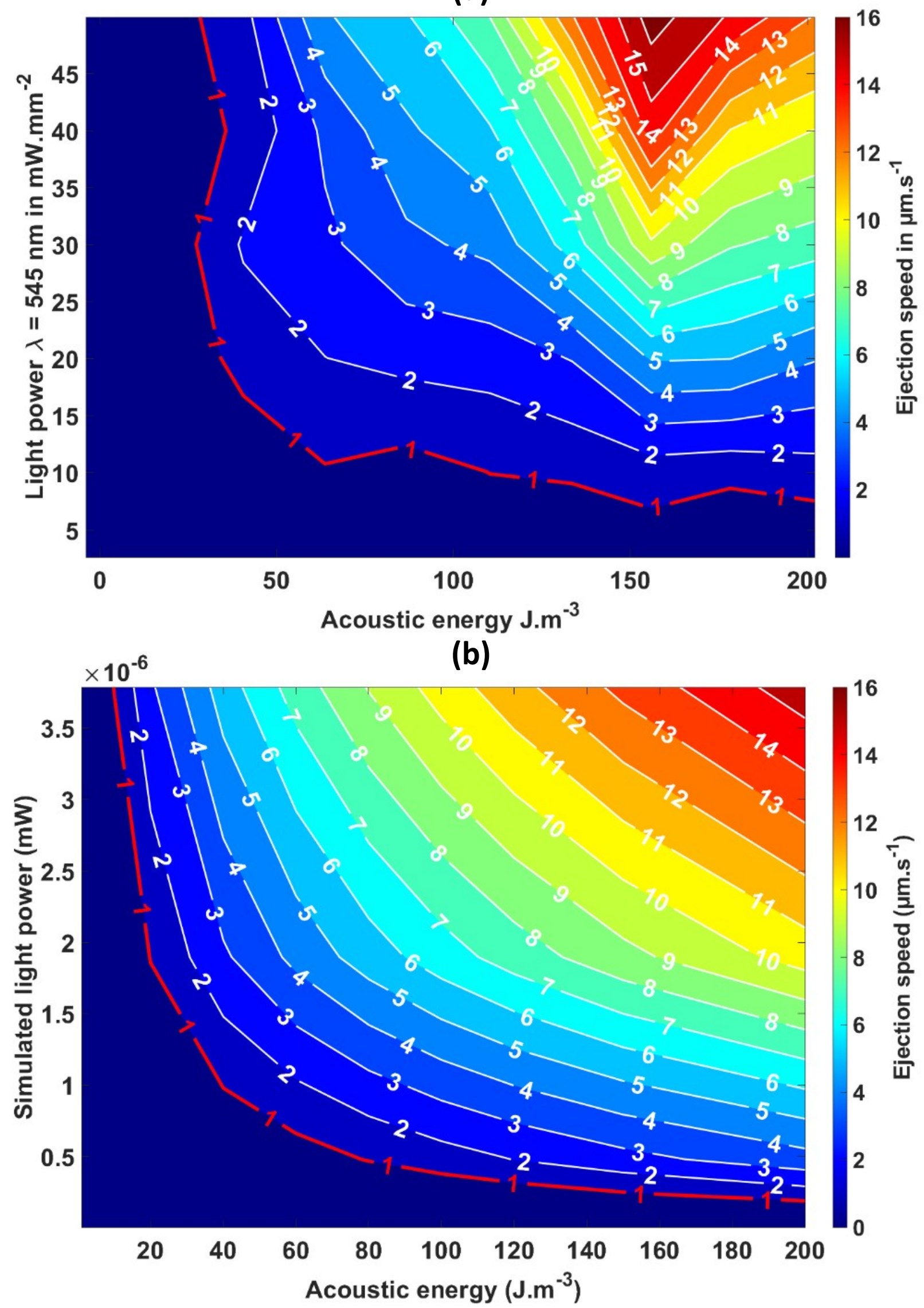
illumination power. It is strikingly similar to the experimental one found $\mathrm{in}^{1}$. The coupled effect of the illumination power and the acoustic energy is well recovered, with a very similar frontier under which no ejection occurs. It proves that our hypothesis are indeed a plausible explanation for the opto-acoustofluidic effect, which would then be related a acousto-thermofludic instability of the fluid.

However, one important step remains unclear, which is the exact mechanism leading to the energy conversion, from the optical power to the eventual heating of the surrounding fluid layer by the particles or aggregate. The simple absorption spectra of the dye or fluorophore contained in the particles do not fit with the specific wavelength for which the expulsion is observed. A more complex coupling with the materials is expected, or a possible effect at the scale of the particle which is both vibrating because of the acoustic wave and absorbing energy injected by the monochromatic illumination.

\section{CONCLUSION}

We have explored in this study the role of thermal effects on the recently discovered "optoacoustofluidic" effect ${ }^{1}$, which leads to the rapid ejection of particles in acoustic levitation when illuminated at specific optical wavelength.

First, we have shown experimentally that for a given acoustic field, optic wave and particle set, the ejection velocity of the particles is decreasing with increasing temperature. For temperatures higher than a critical threshold, the particles or aggregates in acoustic levitation are no longer ejected when illuminated. If this experiment demonstrate that there is 
JASA/Influence of the temperature on the opto-acoustophoretic effect

indeed a thermal effect, it can not be a simple convection effect. Indeed, we verified that the Rayleigh-Bénard instability can not be triggered in our experiment.

We went further using numerical simulation taking into account both thermal effects and acoustic radiation force on the heated fluid. We found out that the introduction of fluid density gradients through the aggregate heating could trigger lateral jets. The numerical simulations demonstrate the existence of a fluid instability, which we qualified as "thermoacoustofluidic". If a fluid is locally heated and, at the same time, undergoes an acoustic radiation force, then a large scale flow is triggered, similar to Rayleigh-Bénard convection rolls but with an inverse rotating motion.

The following hypothesis was then that the particles could be dragged away by the induced flow. Ejection of the particles can thus be understood as a competition between a Stokes drag induced by the fluid motion and the transverse component of the ARF applied to the particles. The simulation yields results that are in very good agreement with the characterization made earlier; especially the coupling between acoustic energy and optical power is recovered leading to a very similar phase diagram as the experimental one ${ }^{1}$.

There are many potential applications for this phenomenon. Indeed, if the postulated heating mechanism is confirmed, then any optically absorbing material set in a standing acoustic field could be turned into a local jet generator when illuminated with the adequate wavelength. Should it be levitating particles or a piece of material set at the right position, the density gradient generated will undergo the acoustic effects and be moved like the particles in our study, allowing for elaborate selective contactless manipulation of particles or cells. 
JASA/Influence of the temperature on the opto-acoustophoretic effect

\section{ACKNOWLEDGMENTS}

We acknowledge the École Doctorale Frontières de l'Innovation en Recherche et Éducation (ED FIRE), Programme Bettencourt, and the Bettencourt-Schueller Foundation for their financial support to Gabriel Dumy's PhD thesis.

\section{REFERENCES}

${ }^{1}$ G. Dumy, M. Hoyos, and J.-L. Aider, "Observation of selective optical manipulation of particles in acoustic levitation," The Journal of the Acoustical Society of America 146(6), $4557-4568$ (2019).

${ }^{2}$ D. Zhou, Y. Gao, J. Yang, Y. C. Li, G. Shao, G. Zhang, T. Li, and L. Li, "Light-ultrasound driven collective "firework" behavior of nanomotors," Advanced Science 5(7), 1800122 (2018).

${ }^{3}$ A. Ashkin, J. M. Dziedzic, J. Bjorkholm, and S. Chu, "Observation of a single-beam gradient force optical trap for dielectric particles," Optics letters 11(5), 288-290 (1986).

${ }^{4}$ A. Ashkin, "History of optical trapping and manipulation of small-neutral particle, atoms, and molecules," IEEE Journal of Selected Topics in Quantum Electronics 6(6), 841-856 (2000) http://ieeexplore.ieee.org/document/902132/ doi: 10.1109/2944.902132.

${ }^{5} \mathrm{~J}$. Wu, "Acoustical tweezers," The Journal of the Acoustical Society of America 89(5), 2140-2143 (1991).

${ }^{6} \mathrm{~J} . \mathrm{Wu}$, "Acoustic radiation force and acoustical tweezers used in biomedical research," The Journal of the Acoustical Society of America 93(4), 2329-2329 (1993). 
${ }^{7}$ G. Whitworth, M. Grundy, and W. Coakley, "Transport and harvesting of suspended particles using modulated ultrasound," Ultrasonics 29(6), 439-444 (1991).

${ }^{8}$ D. Hartono, Y. Liu, P. L. Tan, X. Y. S. Then, L.-Y. L. Yung, and K.-M. Lim, "Onchip measurements of cell compressibility via acoustic radiation," Lab on a Chip 11(23), 4072-4080 (2011).

${ }^{9}$ L. Puskar, R. Tuckermann, T. Frosch, J. Popp, V. Ly, D. McNaughton, and B. R. Wood, "Raman acoustic levitation spectroscopy of red blood cells and plasmodium falciparum trophozoites," Lab on a Chip 7(9), 1125-1131 (2007).

${ }^{10}$ B. R. Wood, P. Heraud, S. Stojkovic, D. Morrison, J. Beardall, and D. McNaughton, "A portable raman acoustic levitation spectroscopic system for the identification and environmental monitoring of algal cells," Analytical chemistry 77(15), 4955-4961 (2005).

${ }^{11}$ K. Chen, M. Wu, F. Guo, P. Li, C. Y. Chan, Z. Mao, S. Li, L. Ren, R. Zhang, and T. J. Huang, "Rapid formation of size-controllable multicellular spheroids via 3d acoustic tweezers," Lab on a Chip 16(14), 2636-2643 (2016).

${ }^{12}$ F. Guo, Z. Mao, Y. Chen, Z. Xie, J. P. Lata, P. Li, L. Ren, J. Liu, J. Yang, M. Dao, et al., "Three-dimensional manipulation of single cells using surface acoustic waves," Proceedings of the National Academy of Sciences 113(6), 1522-1527 (2016).

${ }^{13}$ C. Bouyer, P. Chen, S. Güven, T. T. Demirtaş, T. J. Nieland, F. Padilla, and U. Demirci, "A bio-acoustic levitational (bal) assembly method for engineering of multilayered, 3d brain-like constructs, using human embryonic stem cell derived neuro-progenitors," Advanced Materials 28(1), 161-167 (2016). 
JASA/Influence of the temperature on the opto-acoustophoretic effect

${ }^{14}$ S. Gutiérrez-Ramos, M. Hoyos, and J. Ruiz-Suárez, "Induced clustering of escherichia coli by acoustic fields," Scientific reports 8(1), 1-8 (2018).

${ }^{15}$ A. Garbin, I. Leibacher, P. Hahn, H. Le Ferrand, A. Studart, and J. Dual, "Acoustophoresis of disk-shaped microparticles: A numerical and experimental study of acoustic radiation forces and torques," The Journal of the Acoustical Society of America 138(5), 2759-2769 (2015).

${ }^{16}$ W. Wang, L. A. Castro, M. Hoyos, and T. E. Mallouk, "Autonomous motion of metallic microrods propelled by ultrasound," ACS nano 6(7), 6122-6132 (2012).

${ }^{17}$ S. Ahmed, D. T. Gentekos, C. A. Fink, and T. E. Mallouk, "Self-Assembly of Nanorod Motors into Geometrically Regular Multimers and Their Propulsion by Ultrasound," ACS Nano 8(11), 11053-11060 (2014) https://pubs.acs.org/doi/10.1021/nn5039614 doi: $10.1021 / \mathrm{nn} 5039614$.

${ }^{18}$ S. Ahmed, W. Wang, L. Bai, D. T. Gentekos, M. Hoyos, and T. E. Mallouk, "Density and Shape Effects in the Acoustic Propulsion of Bimetallic Nanorod Motors," ACS Nano 10(4), 4763-4769 (2016) http://pubs.acs.org/doi/10.1021/acsnano.6b01344 doi: 10.1021/ acsnano. $6 \mathrm{~b} 01344$.

${ }^{19}$ D. Ahmed, T. Baasch, B. Jang, S. Pane, J. Dual, and B. J. Nelson, "Artificial Swimmers Propelled by Acoustically Activated Flagella," Nano Letters 16(8), 4968-4974 (2016) http://pubs.acs.org/doi/10.1021/acs.nanolett.6b01601 doi: 10.1021/acs. nanolett.6b01601. 
JASA/Influence of the temperature on the opto-acoustophoretic effect

${ }^{20}$ G. Dumy, N. Jeger-Madiot, X. Benoit-Gonin, T.E. Mallouk, M. Hoyos, and J-L. Aider, "Acoustic manipulation of dense nanorods in microgravity," Microgravity Science and Technology 32(6), 1159-1174 (2020) https://link.springer.com/article/10.1007/ s12217-020-09835-7 doi: 10.1007/s12217-020-09835-7.

${ }^{21}$ I. Leibacher, W. Dietze, P. Hahn, J. Wang, S. Schmitt, and J. Dual, "Acoustophoresis of hollow and core-shell particles in two-dimensional resonance modes," Microfluidics and Nanofluidics 16(3), 513-524 (2014) http://link.springer.com/10.1007/ s10404-013-1240-7 doi: 10.1007/s10404-013-1240-7.

${ }^{22}$ J. Leão-Neto, J. Lopes, and G. Silva, "Core-Shell Particles that are Unresponsive to Acoustic Radiation Force," Physical Review Applied 6(2) (2016) https://link.aps.org/doi/ 10.1103/PhysRevApplied.6.024025 doi: 10.1103/PhysRevApplied.6.024025.

${ }^{23}$ L. P. Gor'kov, "On the forces acting on a small particle in an acoustical field in an ideal fluid," Soviet Physics - Doklady 773-775 (1962).

${ }^{24}$ F. Mitri, "Acoustic radiation force acting on elastic and viscoelastic spherical shells placed in a plane standing wave field," Ultrasonics 43(8), 681-691 (2005) http://linkinghub. elsevier.com/retrieve/pii/S0041624X05000090 doi: 10.1016/j.ultras.2005.03. 002.

${ }^{25}$ M. Settnes and H. Bruus, "Forces acting on a small particle in an acoustical field in a viscous fluid," Physical Review E 85(1) (2012) https://link.aps.org/doi/10.1103/ PhysRevE.85.016327 doi: 10.1103/PhysRevE.85.016327. 
JASA/Influence of the temperature on the opto-acoustophoretic effect

${ }^{26}$ A. A. Doinikov, "Acoustic radiation pressure on a compressible sphere in a viscous fluid," Journal of Fluid Mechanics 267(-1), 1 (1994) http://www.journals.cambridge.org/ abstract_S0022112094001096 doi: 10.1017/S0022112094001096.

${ }^{27}$ G. Dumy, X. Benoit-Gonin, M. Hoyos, and J.-L. Aider, "Acoustic propulsion of metallic nano-cylinders: contribution of the local vertical acceleration from micro to hyper gravity," (2019).

${ }^{28}$ T. Tuziuti, T. Kozuka, and H. Mitome, "Measurement of distribution of acoustic radiation force perpendicular to sound beam axis," Japanese journal of applied physics 38(5S), 3297 (1999).

${ }^{29}$ S. M. Woodside, B. D. Bowen, and J. M. Piret, "Measurement of ultrasonic forces for particle-liquid separations," AIChE journal 43(7), 1727-1736 (1997).

${ }^{30}$ J. T. Karlsen, P. Augustsson, and H. Bruus, "Acoustic Force Density Acting on Inhomogeneous Fluids in Acoustic Fields," Physical Review Letters 117(11) (2016) https: //Iink.aps.org/doi/10.1103/PhysRevLett.117.114504 doi: 10.1103/PhysRevLett. 117.114504

${ }^{31}$ O. Dron and J.-L. Aider, "Acoustic energy measurement for a standing acoustic wave in a micro-channel," EPL (Europhysics Letters) 97(4), 44011 (2012) http://stacks.iop. org $/ 0295-5075 / 97 / i=4 / a=44011$ ?key=crossref .31 b8ebc628f c2d2a9f b8ea61696bb8a0 doi: $10.1209 / 0295-5075 / 97 / 44011$.

${ }^{32}$ N. T. Ouellette, H. Xu, and E. Bodenschatz, "A quantitative study of three-dimensional lagrangian particle tracking algorithms," Experiments in Fluids 40(2), 301-313 (2006). 
JASA/Influence of the temperature on the opto-acoustophoretic effect

583

584

585

586

587

588

589

${ }^{33}$ N. T. Ouellette, "Particle-based measurement techniques for soft matter," Experimental and Computational Techniques in Soft Condensed Matter Physics 180 (2010).

${ }^{34}$ M. Krishnan, V. M. Ugaz, and M. A. Burns, "Pcr in a rayleigh-benard convection cell.(biochemistry)," Science 298(5594), 793-794 (2002).

${ }^{35}$ R. Muddu, Y. A. Hassan, and V. M. Ugaz, "Chaotically accelerated polymerase chain reaction by microscale rayleigh-bénard convection," Angewandte Chemie International Edition 50(13), 3048-3052 (2011). 